\title{
The arithmetic of zero cycles on surfaces with geometric genus and irregularity zero
}

\author{
Kevin R. Coombes ${ }^{\star}$ \\ Department of Mathematics, University of Michigan, Ann Arbor, MI 48109, USA
}

Received May 30, 1990

\section{Introduction}

Let $X$ be a smooth, projective, geometrically irreducible surface over a perfect field $F$. Throughout this paper, it will be assumed that the geometric genus $p_{g}$ and the irregularity $q$ of $X$ both vanish. Denote the separable closure of $F$ by $\bar{F}$. Let $\bar{X}=X \otimes_{F} \bar{F}$ be the surface obtained from $X$ by base extension. It will also be assumed that the group $A_{0}(\bar{X})$ of rational equivalence classes of zero cycles of degree zero on $\bar{X}$ vanishes. This is a technical hypothesis which could presumably be eliminated at the expense of working with $\operatorname{Ker}\left(A_{0}(X) \rightarrow A_{0}(\bar{X})\right)$. For want of a better name, and for ease in stating various results, any surface which satisfies these three hypotheses will be called a pseudo-rational surface.

Bloch [2] has conjectured that the vanishing of $A_{0}(\bar{X})$ should follow from the assumption that $p_{g}=q=0$. This was proven by Bloch, Kas, and Lieberman [5] for all such surfaces which are not of general type; i.e., which have Kodaira dimension less than 2. It has also been proven for particular surfaces of general type by Inose and Mizukami [23], Barlow [1], and Keum [25]. Consequently, the class of pseudo-rational surfaces includes: rational surfaces, Enriques surfaces, elliptic surfaces with $q=0$, the classical Godeaux surface, Burniat-Inoue surfaces, Campedelli surfaces, and the surfaces of Barlow and of Keum.

This paper will study $A_{0}(X)$ for pseudo-rational surfaces defined over fields of number theoretic interest. Bloch [4] introduced $K$-theoretic techniques into the study of zero cycles on rational surfaces. His work was extended $[7,12,15,27,28$, $32,36]$ to achieve a thorough understanding of such cycles. Colliot-Thélène and Raskind [10] developed this machinery further to study codimension two cycles on any variety. The author [14] applied these techniques to Enriques surfaces. Raskind [30] used them to study zero cycles on pseudo-rational surfaces. One of the main results of this paper is a new proof of the following theorem of Raskind [31].

\footnotetext{
* Partially supported by the NSF
} 
Theorem 0.1. Let $X$ be a pseudo-rational surface over a non-archimedean local field $L$ with residue field $F$. Assume that $X$ has very good reduction to a smooth projective surface $Y$ over $F$. Assume also that the characteristic of $F$ is relatively prime to the order of the torsion subgroup of $\mathrm{NS}(\bar{X})$. Then the specialization map on zero cycles defines a natural isomorphism $A_{0}(X) \approx A_{0}(Y)$.

See Sect. 3 for a precise definition of "very good reduction." Since Kato and Saito [24] have computed the group of zero cycles for surfaces over a finite field explicitly, the previous theorem gives a complete computation of the group of zero cycles in the case of very good reduction. Raskind [30] was able to use the local computation to get a finiteness result over number fields, under the restrictive hypothesis that the Galois group acts trivially on the Néron-Severi group. The proof of Theorem 0.1 given here actually produces a somewhat stronger local result. Its statement is rather technical; see Corollary 3.8 for details. This stronger result has the advantage that it can be used to obtain global results without any restrictive hypotheses:

Theorem 0.2. Let $X$ be a pseudo-rational surface over a number field. Then $A_{0}(X)$ is a finite group.

The techniques used here generalize those in [14]. Various complications arise from the Galois action on the Néron-Severi group, but these are dealt with by making the arguments of [14] more "coordinate-free." An obvious question which arises is: are Theorems 0.1 and 0.2 true more generally? In particular, are they true for hyperelliptic surfaces with $A_{0}(X)$ replaced by $\operatorname{Ker}\left(A_{0}(X) \rightarrow A_{0}(\bar{X})\right)$ ? Along these lines, one should consult the work of Gros [19] on ruled surfaces.

The author has been informed that Colliot-Thélène and Raskind, after seeing the results of this paper, have found another proof of Theorem 0.2 . Their methods apparently involve extending Raskind's proof of Theorem 0.1 to the global case, in a manner reminiscent of the finiteness results over global fields of positive characteristic in [13].

\section{Notations and Conventions}

For any finitely generated abelian group $A$, let $A^{D}=\operatorname{Hom}(A, \mathbb{Q} / \mathbb{Z})$ denote its Pontrjagin dual. Given a prime number $l$, let $A\{l\}$ denote the $l$-primary torsion subgroup of $A$. If $A$ is finite of exponent $m$, its Tate twists are defined by $A(n)$ $=A \otimes \mu_{m}^{\otimes n}$. If $A$ is a profinite limit of abelian l-groups, its Tate twists are defined, using the $l$-adic Tate module $\mathbb{Z}_{l}(1)=\lim _{l} \mu_{l}$, to be $A(n)=A \otimes\left(\mathbb{Z}_{l}(1)\right)^{\otimes n}$.

The term surface will, unless otherwise qualified, refer to a smooth, projective, geometrically integral surface over a field $F$. If $E / F$ is any extension field, then $X_{E}$ denotes the surface obtained by base extension, and $E(X)$ denotes the field of $E$-functions of the extended surface. The following combinations of $K$-groups will be used throughout this paper:

$$
\begin{gathered}
Q(E)=K_{2}(E(X)) / K_{2}(E), \\
R(E)=K_{2}(E(X)) / H^{\circ}\left(X_{E}, \mathscr{K}_{2}\right), \\
S(E)=\operatorname{Ker}\left(\coprod_{C \in X_{E}^{1}} E(C)^{*} \rightarrow \coprod_{x \in X_{E}^{2}} \mathbb{Z}\right) .
\end{gathered}
$$


Now assume that $X$ is a surface over $F$. Let $\bar{F}$ be a separable closure of $F$, and let $\bar{X}=X_{\bar{F}}$. The numerical invariants of $\bar{X}$ which are of immediate interest include: the geometric genus, $p_{g}=\operatorname{dim}_{\bar{F}} H^{2}\left(\bar{X}, \mathcal{O}_{\bar{X}}\right)$; the irregularity, $q=\operatorname{dim} \operatorname{Alb}(\bar{X})$; the rank of the Néron-Severi group, $\varrho=\operatorname{rk}(\operatorname{NS}(\bar{X}))$; and the Betti numbers,

$$
B_{i}=\operatorname{dim}_{\mathbb{Q}_{l}} H^{i}\left(\bar{X}, \mathbb{Q}_{l}\right)
$$

for $l$ different from the characteristic of $F$.

Definition. A surface $X$ over a field $F$ will be called pseudo-rational if

(i) $p_{g}=q=0$,

(ii) $\mathrm{CH}_{0}(\bar{X})=\mathbb{Z}$, and

(iii) $\varrho=B_{2}$.

Bloch [2] has conjectured that (i) implies (ii); this is known for many classes of surfaces $[1,5,23,25]$. In characteristic zero, Hodge theory can be used to show that (i) implies (iii). In positive characteristic, (i) also implies (iii) provided one assumes in addition either that $X$ can be lifted to characteristic zero or that $X$ is not of general type [16]. I do not know if (i) implies (iii) for non-liftable surfaces of general type in positive characteristic.

\section{The cohomology of $K_{2}$ and algebraic cycles}

The study of algebraic cycles on rational surfaces has been facilitated by the introduction of $K$-theoretic methods $[4,7,12,15]$. A critical role is played by the cohomology of $\mathscr{K}_{2}$, which can essentially be computed using the Merkurjev-Suslin theorem [26]. Connections between the cohomology of $\mathscr{K}_{2}$ and cycles of codimension two have been explored by Colliot-Thélène and Raskind [10]. The object of this section is to apply their techniques to pseudo-rational surfaces.

The first step in the computation of the cohomology of $\mathscr{K}_{2}$ uses the GerstenQuillen resolution [29]. For any smooth projective variety $X$ defined over a perfect field $F$ and any field extension $E / F$, the groups $R(E)$ and $S(E)$ fit into exact sequences

$$
\begin{gathered}
0 \rightarrow H^{0}\left(X_{E}, \mathscr{K}_{2}\right) \rightarrow K_{2}(E(X)) \rightarrow R(E) \rightarrow 0, \\
0 \rightarrow S(E) \rightarrow \coprod_{C \in X_{E}^{1}} E(C)^{*} \rightarrow \coprod_{x \in X_{E}^{2}} \mathbb{Z} \rightarrow H^{2}\left(X_{E}, \mathscr{K}_{2}\right) \rightarrow 0 .
\end{gathered}
$$

Following the standard convention, $X^{i}$ denotes the set of points of codimension $i$ on the scheme $X$. There is another exact sequence relating these groups:

$$
0 \rightarrow R(E) \rightarrow S(E) \rightarrow H^{1}\left(X_{E}, \mathscr{K}_{2}\right) \rightarrow 0 .
$$

Now assume that $X$ is a pseudo-rational surface. The cohomology of $\mathscr{K}_{2}$ over an algebraically closed field has been completely computed. The characteristic zero and prime-to- $p$ parts in characteristic $p$ are due to Colliot-Thélène and Raskind [10]. The p-part is due to Gros and Suwa [20] using results of [36]. By definition, pseudo-rational surfaces have Chow group $\mathrm{CH}_{0}(\bar{X})=H^{2}\left(\bar{X}, \mathscr{K}_{2}\right)=\mathbb{Z}$. Thus, their results ([10, Proposition 1.14, Theorem 2.12, and Proposition 2.15; and [20, Theorem V.2.3 and Theorem V.3.6]) are summarized by the exact sequences

$$
\begin{gathered}
\left.0 \rightarrow K_{2}(\bar{F}) \rightarrow H^{0}\left(\bar{X}, \mathscr{K}_{2}\right)\right) \rightarrow \bigoplus_{l \neq p} H^{2}\left(\bar{X}, \mathbb{Z}_{l}(2)\right)\{l\} \rightarrow 0, \\
0 \rightarrow U \rightarrow \operatorname{Pic}(\bar{X}) \otimes \bar{F}^{*} \rightarrow H^{1}\left(\bar{X}, \mathscr{K}_{2}\right) \rightarrow \bigoplus_{l} H^{3}\left(\bar{X}, \mathbb{Z}_{l}(2)\right)\{l\} \rightarrow 0,
\end{gathered}
$$


where $U$ is a uniquely divisible group. In fact, as Colliot-Thélène has pointed out, $U=0$. This follows since the composite

$$
\operatorname{Pic}(\bar{X}) \otimes \bar{F}^{*} \rightarrow H^{1}\left(\bar{X}, \mathscr{K}_{2}\right) \rightarrow \operatorname{Hom}\left(\operatorname{Pic}(\bar{X}), \bar{F}^{*}\right),
$$

where the last arrow comes from Weil reciprocity, has finite kernel.

Now, the sums of étale cohomology groups in both (1.4) and (1.5) are always finite. When $X$ is a pseudo-rational surface, let $P$ be the torsion subgroup of $\operatorname{Pic}(\bar{X})$. An analysis of the Kummer sequence and an application of Poincare duality shows that

$$
\oplus_{l} H^{2}\left(\bar{X}, \mathbb{Z}_{l}(2)\right)\{l\} \approx P(1), \text { and } \oplus_{l} H^{3}\left(\bar{X}, \mathbb{Z}_{l}(2)\right)\{l\} \approx P^{D}(1) .
$$

If the characteristic $p$ is positive, two points should be noted. First, the quotient in sequence (1.4) only consists of the prime-to- $p$ part of $P(1)$, which will be denoted by $P(1)^{\prime}$. Second, the quotient in sequence (1.5) includes the factor $H^{3}\left(\bar{X}, \mathbb{Z}_{p}(2)\right)\{p\}$, which is defined using the logarithmic de Rham-Witt complex. Consult [20] for details.

The first task of this section is to develop a better understanding of the interesting elements in (1.4). Let $m$ be the exact exponent of the finite abelian group $P(1)^{\prime}$. An element of $P(1)^{\prime}=P \otimes \mu_{m}$ can always be written in the form $d \otimes \zeta$, where $d \epsilon P$ and $\zeta$ is an $m^{\text {th }}$ root of unity. Now let $d=[D]$ be the class of a torsion divisor $D$. Choose a function $f \in \bar{F}(X)^{*}$ with divisor $(f)=m D$.

Lemma 1.6. The assignment $[D] \otimes \zeta \mapsto\{f, \zeta\} \in K_{2}(\bar{F}(X))$ defines a homomorphism of Galois modules which splits the exact sequence (1.4). In particular,

$$
H^{0}\left(\bar{X}, \mathscr{K}_{2}\right) \approx K_{2}(\bar{F}) \oplus P(1)^{\prime} .
$$

Proof. See also $[10,36]$. Notice first that the diagonal action of $\mathrm{Gal}(\bar{F} / F)$ on the tensor product $P(1)^{\prime}$ is clearly compatible under this assignment with its action on $K_{2}(\tilde{F}(X))$.

To see that the assignment is independent of the choice of $f$, let $\lambda \in \bar{F}^{*}$ be a constant. By bilinearity, $\{\lambda f, \zeta\}=\{\lambda, \zeta\}\{f, \zeta\}$. But $\{\lambda, \zeta\}=\left\{\sqrt[m]{\lambda}, \zeta^{m}\right\}=1$. Further, if $D$ is replaced by a linearly equivalent divisor $D^{\prime}$, then $f$ can be replaced by $f^{\prime}=f g^{m}$ where $(g)=D^{\prime}-D$. Then

$$
\left\{f^{\prime}, \zeta\right\}=\left\{f g^{m}, \zeta\right\}=\{f, \zeta\}\left\{g, \zeta^{m}\right\}=\{f, \zeta\} .
$$

Hence, this assignment actually gives a well-defined map to $K_{2}(\bar{F}(X))$. It is a homomorphism since the Steinberg symbol is bilinear, and if $(f)=m D,(g)=m E$, then $(f g)=m D+m E$.

It remains to show that this homomorphism splits the exact sequence. Since $P(1)^{\prime}$ is finite, it is enough to check that the elements $\{f, \zeta\}$ actually lie in $H^{0}\left(\bar{X}, \mathscr{K}_{2}\right)$ and survive in the quotient $P(1)^{\prime}$. Suslin's computation of torsion [36] shows that $\{f, \zeta\}$ is non-trivial whenever $f$ is not an $m^{\text {th }}$ power, which will be the case if $(f)=m D$ and $D$ represents a non-trivial divisor class. Let $D=\sum n_{C} C$ where $C$ ranges through the irreducible divisors. Applying the tame symbol $T_{C}$ associated to $C$ gives

$$
T_{C}(\{f, \zeta\})=\zeta^{m n_{C}}=1 .
$$

Thus, $\{f, \zeta\} \in H^{\mathbf{0}}\left(\bar{X}, \mathscr{K}_{2}\right)$. Finally, since $\{f, \zeta\}$ is torsion, unique divisibility implies that $\{f, \zeta\}$ is not an element of $K_{2}(\bar{F})$. The result follows. 
When $X$ is a pseudo-rational surface over an arbitrary field $F$, the Galois group $G=\operatorname{Gal}(\bar{F} / F)$ acts on the $K$-cohomology. The relations with zero cycles are mediated by this action.

Lemma 1.7. $S(\bar{F})^{G}=S(F)$ and $H^{1}(G, S(\bar{F}))=A_{0}(X)$.

Proof. See also [10]. By definition, the Chow group of zero cycles of degree zero is trivial on a pseudo-rational surface over $\bar{F}$. So, the Galois cohomology of (1.2) is

$$
0 \rightarrow S(\bar{F})^{G} \rightarrow \coprod F(D)^{*} \rightarrow(\coprod \mathbb{Z})^{0} \rightarrow H^{1}(G, S(\bar{F})) \rightarrow 0,
$$

where the first direct sum is over all irreducible curves $D$ on $X$, while the second is over all closed points and the superscript 0 designates the subgroup of elements of degree zero. However, one also has an exact sequence

$$
0 \rightarrow S(F) \rightarrow \amalg F(D)^{*} \rightarrow(\amalg \mathbb{Z})^{0} \rightarrow A_{0}(X) \rightarrow 0 .
$$

The result follows by comparing these two exact sequences.

For any Galois extension field $E / F$, define $Q(E)=K_{2}(E(X)) / K_{2}(E)$. From (1.1), (1.4) and the definition of $Q$, one has an exact sequence

$$
0 \rightarrow P(1)^{\prime} \rightarrow Q(\bar{F}) \rightarrow R(\bar{F}) \rightarrow 0 .
$$

Assuming either that $X$ has a smooth zero cycle of degree one or that the cohomological dimension of the base field is at most 1 , Colliot-Thélène [7] has shown that $H^{0}(G, Q(E))=Q(F)$ and $H^{1}(G, Q(E))=0$.

Proposition 1.9. Let $X$ be a pseudo-rational surface over a field $F$. Assume either that $X$ has a smooth zero cycle of degree one, or that $c d(F)=1$. Then there is an exact diagram

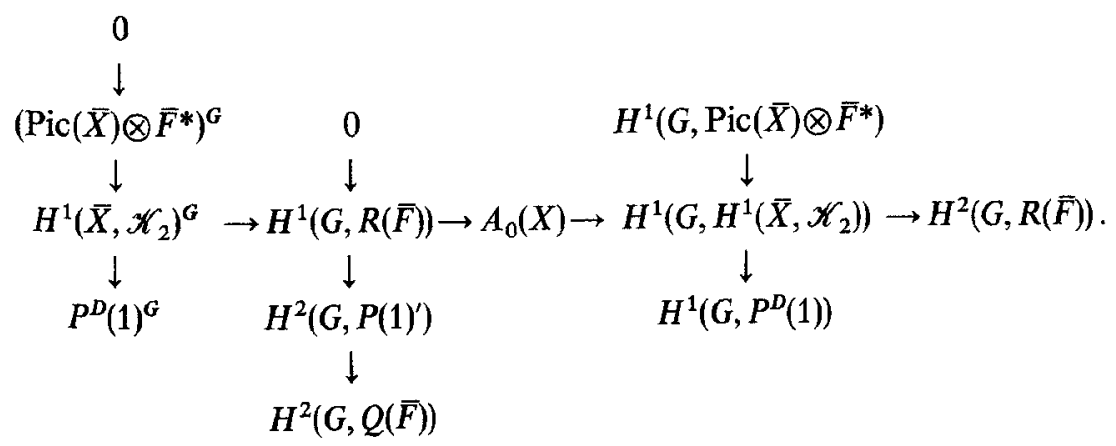

Proof. The horizontal row is the cohomology of sequence (1.3), using Lemma 1.7. The left and right vertical columns are the cohomology of sequence (1.5). The remaining column is the cohomology of sequence (1.8).

To illustrate how these results may be applied, a bound is derived on the number of real components of a pseudo-rational surface over $\mathbb{R}$. (One should be aware, however, that a better bound has been obtained [9] by other methods.) The idea that the present computations could be combined with the work of ColliotThélène and Ischebeck [8] to obtain some bound was suggested, in the context of Enriques surfaces, by Colliot-Thélène. To state the bound, define the 2-rank of a finitely generated abelian group $A$ to be the dimension of the $\mathbb{Z} / 2$-vector space 
$A\{2\} / 2 A\{2\}$. Now let $\varrho$ be the rank of $\operatorname{Pic}(X)$ and let $\tau$ be the 2-rank of its torsion subgroup.

Theorem 1.10. Let $X$ be a pseudo-rational surface over $\mathbb{R}$. Then $X(\mathbb{R})$ has at most $\varrho+2 \tau$ connected components.

Proof. One may clearly assume that $X(\mathbb{R})$ is non-empty. Then, by [8], the group $A_{0}(X)$ is isomorphic to $(\mathbb{Z} / 2)^{s-1}$ where $s$ is the number of connected components. By Proposition 1.9, the size of $A_{0}(X)$ is bounded by the sum of the sizes of the three cohomology groups $H^{2}(G, P(1)), H^{1}\left(G, P^{D}(1)\right)$, and $H^{1}\left(G, \operatorname{Pic}\left(X_{\mathbb{C}}\right) \otimes \mathbb{C}^{*}\right)$. Since $G=\mathbb{Z} / 2$, all three cohomology groups are $\mathbb{Z} / 2$-vector spaces. Since the 2-ranks of $P^{D}(1)$ and $P(1)$ are both equal to $\tau$, each of the first two cohomology groups has 2-ranks bounded by $\tau$.

Write $N=\operatorname{Pic}\left(X_{\mathscr{C}}\right) / P$ for the group of numerical equivalence classes of divisors on $X$. Since $\mathbb{C}^{*}$ is divisible, $\operatorname{Pic}\left(X_{\mathbb{C}}\right) \otimes \mathbb{C}^{*}$ is isomorphic to $N \otimes \mathbb{C}^{*}$. Let $\sigma$ be the nontrivial element of $G$. Then $\sigma$ acts on the finitely generated free abelian group $N$ through a unimodular integral matrix. So, $\sigma$ is a sum of representations of the form $(-1),(1)$, and $\left(\begin{array}{ll}0 & 1 \\ 1 & 0\end{array}\right)$. By explicit computation, $H^{1}\left(G, N \otimes \mathbb{C}^{*}\right)$ is a $\mathbb{Z} / 2$-module with rank equal to the number of $(-1)$ factors. Since the rank of the Picard group is $\varrho$ and since complex conjugation cannot act on the entire Picard group by $\sigma(x)=-x$, the number of $(-1)$ factors is at most $\varrho-1$. The result follows.

\section{Maximal unramified extensions of local fields}

The next major goal is to compute the group of zero cycles on a pseudo-rational surface over a $p$-adic field $L$. It will, however, be necessary first to consider auxiliary fields - in particular, both the finite residue field and the maximal unramified extension field of $L$ play significant roles.

Proposition 2.1. If $X$ is a pseudo-rational surface over a finite field $F$ whose characteristic is relatively prime to the order of $P$, then there is an isomorphism

$$
\gamma: A_{0}(X) \rightarrow H^{1}\left(G, H^{1}\left(\bar{X}, \mathscr{K}_{2}\right)\right) \rightarrow H^{1}\left(G, P^{D}(1)\right) .
$$

Proof. See [11] for a more general result. Kato and Saito [24] have shown that $A_{0}(X) \approx \operatorname{Hom}_{G}(\mu, P)^{*}$. But this group has the same order as $H^{1}\left(G, P^{D}(1)\right)$. So, $\gamma$ is a map between two groups of the same order. It suffices, therefore, to show that $\gamma$ is injective. This will follow from chasing the diagram in Proposition 1.9: Since $c d(F)=1$, one has $H^{2}\left(G, P(1)^{\prime}\right)=0$. Moreover, the first cohomology group of the torus $\operatorname{Pic}(\bar{X}) \otimes \bar{F}^{*}$ also vanishes over a finite field.

Definition 2.2. Let $X$ be a surface over an arbitrary field $F$. An extension field $E / F$ will be called a splitting field for $X$ provided the Néron-Severi group $\operatorname{NS}(\bar{X})$ is generated by $E$-rational curves.

Lemma 2.3. Every surface has a splitting field of finite degree.

Proof. Choose a finite set of curves defined over $\bar{F}$ which generates $\operatorname{NS}(\bar{X})$. Since each curve is already defined over a finite extension, the whole collection of generators is defined over a single finite extension. 
Proposition 2.4. Let $X$ be a surface over a p-adic field L. Assume that $X$ has good reduction, that $H^{1}\left(X, \mathcal{O}_{X}\right)=0$, and that $\mathrm{NS}(\bar{X})$ has no $p$-torsion. Then $X$ has a finite unramified splitting field.

Proof. Take a smooth proper model of $X$ over the ring of integers in $L$. Let $F^{\prime} / F$ be a finite splitting extension of the closed fibre. Let $E^{\prime} / L$ be the unique unramified extension with residue field $F^{\prime}$. Away from $p$, the Néron-Severi group injects into the second étale cohomology group, and the étale cohomology is independent of smooth fibres. So, the Galois group $\operatorname{Gal}(\bar{L} / L)$ acts on $\operatorname{NS}(\bar{X})$ through its unramified quotient. In particular, $\operatorname{Gal}\left(\bar{L} / E^{\prime}\right)$ acts trivially on $\operatorname{NS}(\bar{X})$. Without loss of generality, one may assume $L=E^{\prime}$.

Write $G=\operatorname{Gal}(\bar{L} / L)$. The exact sequence of low degree terms in the Serre spectral sequence for $\mathbb{G}_{m, X}$ gives

$$
0 \rightarrow \operatorname{Pic}(X) \rightarrow \operatorname{Pic}(\bar{X})^{G} \rightarrow \operatorname{Br}(L) .
$$

Let $B$ denote the image in the Brauer group of the final map. Since $L$ is local, $B$ is cyclic and must necessarily be split by an unramified extension $E / L$. However, the vanishing of $H^{1}\left(X, \mathcal{O}_{X}\right)$ implies that $\mathrm{Pic}(X)=\mathrm{NS}(X)$. Moreover, the same equality holds over the algebraic closure. Since $G$ acts trivially on $\operatorname{NS}(\bar{X})$, the extension $E / L$ is a finite unramified splitting extension for $X$.

For the remainder of this section, let $W$ denote the maximal unramified extension of a $p$-adic field. The residue field $\bar{F}$ of $W$ is isomorphic to the algebraic closure of a finite field. Write $I=\operatorname{Gal}(\bar{W} / W)$. It will eventually be necessary to compute Galois cohomology for the action of $I$ on various modules related to the $K$-cohomology of a pseudo-rational surface. Repeated use will be made of the fact that $W$ has cohomological dimension 1.

Lemma 2.5. Let $X$ be a pseudo-rational surface over $W$. Then $H^{i}\left(I, \operatorname{Pic}(\bar{X}) \otimes \bar{W}^{*}\right)=0$ for all $i>0$.

Proof. The result follows after making a finite splitting extension from Hilbert's Theorem 90 and $c d(W)=1$. By the Serre spectral sequence, the higher cohomology is torsion, with bounded exponent. But the Kummer sequence shows that the higher cohomology is divisible. Therefore, it vanishes.

Lemma 2.6. Let $X$ be a pseudo-rational surface over $W$. Then

$$
H^{2}\left(I, H^{1}\left(\bar{X}, \mathscr{K}_{2}\right)\right)=0, \quad H^{1}\left(I, H^{1}\left(\bar{X}, \mathscr{K}_{2}\right)\right) \approx H^{1}\left(I, P^{D}(1)\right),
$$

and there is an exact sequence

$$
0 \rightarrow\left(\operatorname{Pic}(\bar{X}) \otimes \bar{W}^{*}\right)^{I} \rightarrow H^{1}\left(\bar{X}, \mathscr{K}_{2}\right)^{I} \rightarrow P^{D}(1)^{I} \rightarrow 0 .
$$

Proof. Use $c d(W)=1$ and Lemma 2.5 to compute the cohomology of sequence (1.5).

Lemma 2.7. Let $X$ be a pseudo-rational surface over $W$. Then $H^{1}(I, R(\bar{W}))=0$ and $H^{2}(I, R(\bar{W})) \approx H^{2}(I, Q(\bar{W}))$.

Proof. Since $c d(W) \leqq 1$, one may use Colliot-Thélène's result [7] that $H^{1}(I, Q(\bar{W}))=0$. Now take the cohomology of sequence (1.8).

Proposition 2.8. Let $X$ be a pseudo-rational surface over $W$. Then

(i) $H^{0}\left(X, \mathscr{K}_{2}\right)=K_{2}(W) \oplus P(1)^{I}$. 
(ii) There is an exact sequence

$$
\left.0 \rightarrow H^{1}(I, P(1)) \rightarrow H^{1}\left(X, \mathscr{K}_{2}\right) \rightarrow H^{1}\left(\bar{X}, \mathscr{K}_{2}\right)\right)^{I} \rightarrow 0 .
$$

Proof. Again using [7], the Galois cohomology of sequence (1.8) is

$$
0 \rightarrow P(1)^{I} \rightarrow Q(W) \rightarrow R(\bar{W})^{I} \rightarrow H^{1}(I, P(1)) \rightarrow 0 .
$$

Using the Galois splitting of Lemma 1.6, one obtains a commutative diagram

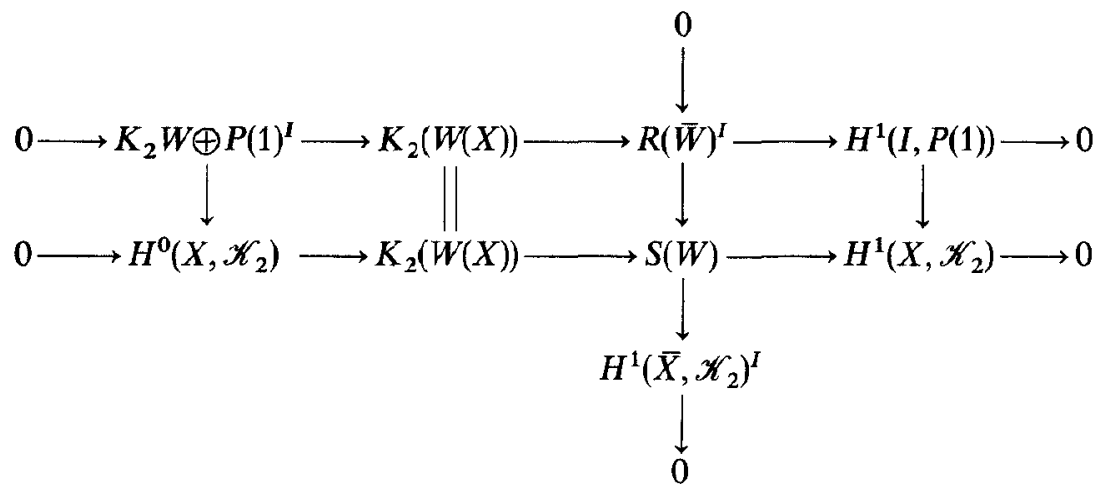

where the vertical sequence is the Galois invariants of (1.3); surjectivity follows from Lemma 2.7. The lemma now follows from a straightforward diagram chase.

Corollary 2.9. There are exact sequences

$$
\begin{gathered}
0 \rightarrow P(1)^{I} \rightarrow Q(W) \rightarrow R(W) \rightarrow 0, \\
0 \rightarrow R(W) \rightarrow R(\bar{W})^{I} \rightarrow H^{1}(I, P(1)) \rightarrow 0 .
\end{gathered}
$$

Proof. This is another interpretation of Proposition 2.8 in terms of the cohomology of (1.8).

Lemma 2.10. If $X$ is a pseudo-rational surface over $W$, then there is a natural injection

$$
\gamma: A_{0}(X) \rightarrow H^{1}\left(I, H^{1}\left(\bar{X}, \mathscr{K}_{2}\right)\right) \rightarrow H^{1}\left(I, P^{D}(1)\right) .
$$

Proof. Use Proposition 1.9, Lemma 2.5, and Lemma 2.7.

The principal task of this section is to provide a concrete geometric description of $\gamma$. Note first that the cup-product

$$
H^{1}\left(I, P^{\mathbf{D}}(1)\right) \otimes H^{\mathbf{0}}(I, P) \rightarrow H^{1}\left(I, \mu_{m}\right) \approx W^{*} / W^{* m} \approx \mathbb{Z} / m
$$

is a duality pairing. So, given a zero cycle $z$ of degree zero, it is enough to describe how to pair $\gamma(z)$ against Galois-invariant, torsion elements in the Picard group. This duality pairing, in turn, is induced by a pairing on $K$-cohomology.

Let $\bar{X}$ be a smooth, projective, geometrically integral surface over an algebraically closed field $k$. There is a natural pairing

$$
\langle,\rangle: H^{1}\left(\bar{X}, \mathscr{X}_{2}\right) \otimes \operatorname{Pic}(\bar{X}) \rightarrow H^{2}\left(\bar{X}, \mathscr{K}_{3}\right) \rightarrow \bar{k}^{*} .
$$


There are two approaches to the definition of such a pairing. One is via the formal properties of algebraic $K$-theory. The other is to define a pairing explicitly on generators of $\operatorname{Pic}(\bar{X})$ and $H^{1}\left(\bar{X}, \mathscr{K}_{2}\right)$ which "meet properly," and to extend the definition via the moving lemma. That these two approaches coincide in the case of intersecting algebraic cycles is a theorem of Grayson [18]. The approach taken here is modelled on Gillet's second proof [17] of Grayson's theorem. First, formal properties of $\boldsymbol{K}$-theory will be used to define the pairing. Next, Bertini's theorem will be used to move representatives of $K$-cohomology classes into general position. Finally, the projection formula will be used to get an explicit formula for the pairing when the representatives are in general position.

The first map in (2.11) is defined to be the pairing on cohomology induced by the Waldhausen product [39] in algebraic $K$-theory. Now let $s: \bar{X} \rightarrow \bar{k}$ be the structure map. Since $\bar{X}$ is a surface, the edge homomorphism $\varepsilon$ in the BrownGersten-Quillen spectral sequence can be composed with $s_{*}$ to get maps

$$
H^{2}\left(\bar{X}, \mathscr{K}_{q}\right)=E_{2}^{2,-q} \rightarrow E_{o 0}^{2,-q} \stackrel{\varepsilon}{\longrightarrow} K_{q-2}(\bar{X}) \stackrel{s_{*}}{\longrightarrow} K_{q-2}(\bar{W}) .
$$

The second map in (2.11) is defined to be the special case of this composite when $q=3$. By abuse of notation, it will also be denoted $s_{*}$.

Lemma 2.12. Let $\alpha \in H^{1}\left(\bar{X}, \mathscr{K}_{2}\right)$ and let $Y \subset \bar{X}$ be an irreducible curve. Let $\tilde{X}$ be obtained byblowing up points in $\bar{X}$ until the proper transform $\tilde{Y}$ of $Y$ is non-singular. Let $j$ denote the canonical composite $\widetilde{Y} \hookrightarrow, \bar{X} \rightarrow X$. Then $\langle\alpha,[Y]\rangle=(s j)_{*}\left(j^{*}(\alpha)\right)$.

Proof. The class $[Y] \in \operatorname{Pic}(\bar{X})$ is nothing other than $j_{*}[\tilde{Y}]$ where $[\tilde{Y}] \in H^{0}\left(\widetilde{Y}, \mathscr{K}_{0}\right) \approx \mathbb{Z}$ is the canonical generator. This claim follows from the standard descriptions of how the Picard group changes under blowups; see, for example, [22].

As in [17], given any scheme $X$ and any $q \geqq 0$, one can define complexes $I_{X}^{*}(q)$ of flasque sheaves in the Zariski topology by

$$
\Gamma\left(U, I_{X}^{p}(q)\right)=E_{1}^{p-q,-q}(U)=\prod_{x \in U^{p}-q} K_{2 q-p}(k(x)) .
$$

Since these sheaves are flasque, there are canonical isomorphisms

$$
\mathbb{H}^{n}\left(X, I_{X}^{*}(q)\right) \approx H^{n-q}\left(X, \mathscr{K}_{q}\right)
$$

between hypercohomology and $K$-cohomology whenever $X$ is regular of finite type over a field. In his proof of the proposition on p. 410 of [17], Gillet shows that if $f: Z \rightarrow X$ is a proper morphism of smooth varieties over a field, and if $r=\operatorname{dim}(X)$ $-\operatorname{dim}(Z)$, then for every $p$ and $q$ there is a commutative diagram

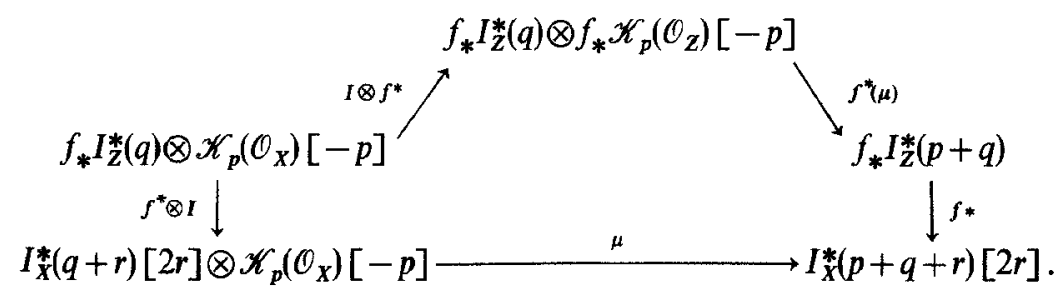

The map $\mu$ induces the Waldhausen product on $K$-theory.

Apply this result to the map $j: \widetilde{Y} \rightarrow \bar{X}$ (so that $r=1$ ) in the case $q=0, p=2$. Now take cohomology, using the fact that $j$ is a finite morphism. One obtains a 
commutative diagram

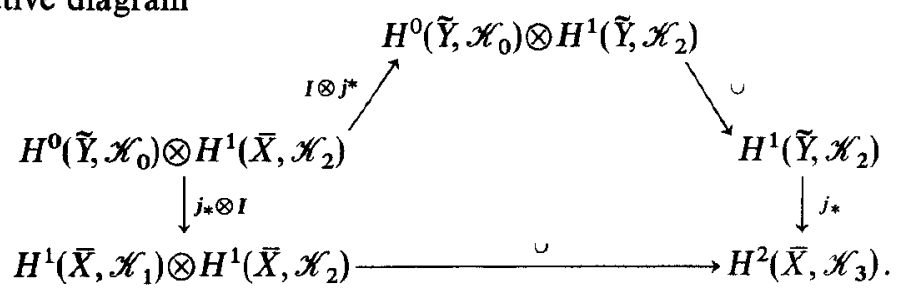

Since $[\tilde{Y}]$ is the unit element in the ring $\oplus H^{p}\left(\widetilde{Y}, \mathscr{K}_{q}\right)$, the commutativity of the last diagram implies

Therefore,

$$
j_{*}[\tilde{Y}] \cup \alpha=j_{*}\left([\tilde{Y}] \cup j^{*}(\alpha)\right)=j_{*}\left(j^{*}(\alpha)\right) .
$$

$$
\langle\alpha,[Y]\rangle=s_{*}(\alpha \cup[Y])=s_{*}\left(\alpha \cup j_{*}[Y]\right)=s_{*}\left(j_{*}\left(j^{*}(\alpha)\right)\right)=(s j)_{*}\left(j^{*}(\alpha)\right) \text {. }
$$

Let $\alpha \in H^{1}\left(\bar{X}, \mathscr{K}_{2}\right)$ and $\beta \in \operatorname{Pic}(\bar{X})$. The next step in finding an explicit description of $\langle\alpha, \beta\rangle$ is to pick convenient representatives for these cohomology classes. Some more notation is needed. If $C$ is an irreducible curve on $\bar{X}$ and if $h \in \bar{W}(C)^{*}$ is any function on that curve, let $[C, h)$ denote the corresponding element of $\coprod_{C} \bar{W}(C)^{*}$. Now let $a=\sum_{c}\left[C, h_{C}\right) \in S(\bar{W})$ be a representative of $\alpha$. (One should recall from the notations and conventions that $a$ lives in $S(\bar{W})$ and represents a class in $H^{1}\left(\bar{X}, \mathscr{K}_{2}\right)$ if and only if $\sum_{C} \operatorname{div}\left(h_{C}\right)=0$.) The support of $a$, written $\operatorname{Supp}(a)$, is defined to be the finite union of those curves $C$ such that $h_{C} \neq 1$. Also define $\operatorname{Sing}(a)$ to be the finite set of singular points on $\operatorname{Supp}(a)$. Notice that, since the divisors $\operatorname{div}\left(h_{c}\right)$ cancel, $\operatorname{Sing}(a)$ must contain all points in the support of those divisors. Now say that a smooth curve $Y \in \bar{X}$ meets a properly if $Y$ does not pass through any of the points of $\operatorname{Sing}(a)$ and if $Y$ intersects each curve in $\operatorname{Supp}(a)$ transversally. Begin with any representative $B$ of $\beta$. Choose an ample divisor $H$ on $\bar{X}$, and an integer $n$ such that $n H$ and $B+n H$ are both very ample. By Bertini's theorem, one can choose representatives of the linear equivalence classes $|n H|$ and $|B+n H|$ which are irreducible, nonsingular, and meet $a$ properly. Thus, it suffices to compute $\langle[a],[Y]\rangle$ for a smooth, very ample curve $Y$ which meets $a$ properly.

Let $j: Y \rightarrow \bar{X}$ be the canonical closed immersion, and let $s: \bar{X} \rightarrow \bar{k}$ be the structure map. So, sj: $Y \rightarrow \bar{k}$ is the structure map for $Y$. Thus, the composite

$$
\coprod_{y \in Y^{1}} k(y)^{*} \rightarrow H^{1}\left(Y, \mathscr{K}_{2}\right) \stackrel{(s j) *}{\longrightarrow} k^{*}
$$

is given by multiplying together the canonical maps $k(y)^{*} \rightarrow k^{*}$. Therefore, to complete the description of the pairing, it is enough, by Lemma 2.12 , to give an explicit description of $j^{*}: H^{1}\left(\bar{X}, \mathscr{K}_{2}\right) \rightarrow H^{1}\left(Y, \mathscr{K}_{2}\right)$. If $y \in C \cap Y$, let $l_{y}(C, Y)$ denote the intersection multiplicity. By transversality, $\imath_{y}(C, Y)$ is always either 0 or 1 . Write $C \cdot Y=\sum_{y} \imath_{y}(C, Y) y$.

Lemma 2.13. Let $a=\sum_{c}\left[C, h_{C}\right)$ be an element of $S(\bar{W})$ and let $j: Y \hookrightarrow \bar{X}$ be the inclusion of an irreducible, nonsingular, very ample curve which meets a properly. Then

$$
j^{*}(a)=j^{*}\left(\sum_{c}\left[C, h_{c}\right)\right)=\sum_{y \in Y^{1}}\left(\prod_{C \ni y} h_{c}(y)^{x_{y}(C, r)}\right) .
$$


Proof. Let $U=\bar{X} \backslash \operatorname{Sing}(a)$. Since $Y$ meets $a$ properly, $U$ is an open neighborhood of $Y$ in $\bar{X}$. Since the complement of $U$ in $\bar{X}$ has codimension 2, the pullback $H^{1}\left(\bar{X}, \mathscr{K}_{2}\right) \rightarrow H^{1}\left(U, \mathscr{K}_{2}\right)$ is injective (see [35, Theorem 2.1]). Without loss of generality, then, $\bar{X}$ can be replaced by $U$. More importantly, the curves in the support of $a$ become smooth curves $C$ on which each $\operatorname{div}\left(h_{C}\right)$ is identically zero. Thus, $h_{C}$ actually lives in $H^{0}\left(C, \mathscr{K}_{1}\right)$. Moreover, the class of $\left[C, h_{C}\right)$ in $H^{1}\left(U, \mathscr{K}_{2}\right)$ is equal to $i_{*}\left(h_{C}\right)$ where $i: C \rightarrow U$ is the canonical inclusion.

Write $Z=C \cap Y$. There is a commutative square

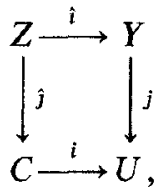

which is a pullback in the category of schemes. All the maps are closed immersions; $Z$ is a finite set of points; $C$ and $Y$ are smooth curves; and $U$ is a smooth quasiprojective surface. Since $\hat{j}^{*}$ is given by evaluating functions on $C$ at points of $Z$, it is enough to verify that the following square commutes:

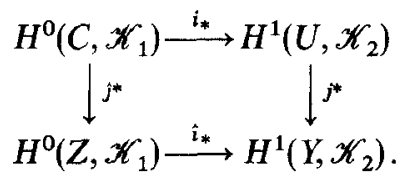

To verify commutativity, define $F^{1}=\operatorname{Ker}\left(K_{1}(U) \rightarrow H^{0}\left(U, \mathscr{K}_{1}\right)\right)$; this is one of the steps in the filtration on $K$-theory coming from the abutment of the BrownGersten-Quillen spectral sequence. Now consider the diagram

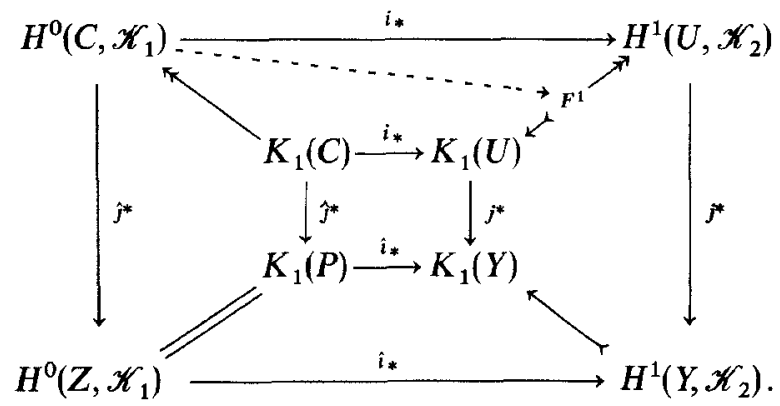

The small central square commutes by Proposition 2.11 on p. 127 of [29]. The lower trapezoid and the upper trapezoid commute because the Brown-GerstenQuillen spectral sequence is covariant for proper morphisms. The proofs of commutativity for the remaining trapezoids are similar, so only the proof for the right-hand-side is provided. The first step is to show that the composite $F^{2}=H^{2}\left(U, \mathscr{K}_{3}\right) \rightarrow F^{1} \rightarrow K_{1}(U) \rightarrow K_{1}(Y)$ is zero. But $H^{2}\left(U, \mathscr{K}_{3}\right)$ is generated by the images of $K_{1}(k(x))$ as $x$ ranges through the closed points of $U \backslash Y$, and the composite

$$
K_{1}(k(x)) \stackrel{\left(i_{x}\right)_{*}}{\longrightarrow} K_{1}(U) \stackrel{j^{*}}{\longrightarrow} K_{1}(Y)
$$

is clearly zero. Consequently, there is an induced map $H^{1}\left(U, \mathscr{K}_{2}\right)=F^{1} / F^{2} \rightarrow K_{1}(Y)$. Now it is enough to show that the composite

$$
F^{1} \rightarrow H^{1}\left(U, \mathscr{K}_{2}\right) \rightarrow K_{1}(Y) \rightarrow H^{0}\left(Y, \mathscr{K}_{1}\right) \hookrightarrow K_{1}(\bar{k}(Y))
$$


is also zero. But elements of $F^{1}$ come from coherent sheaves on $U$ whose support has codimension 1 . The only potential difficulty comes from sheaves supported on $Y$. On the one hand, a standard computation of Tor-groups (see, for example, Proposition 12 of [6]) shows that $i^{*} i_{*}$ is equal to the difference of the maps induced by the functors $\left[\mathcal{O}_{Y} \otimes \cdot\right]$ and $\left[\mathcal{O}_{Y}(-Y) \otimes \cdot\right]$. On the other hand, since $Y$ is very ample, one can choose a smooth curve $Y_{1}$ which is linearly equivalent to $Y$ and intersects it properly in a subscheme $T=Y \cap Y_{1}$. Apply the exactness theorem of [29] to the functors defined by tensoring with the exact sequence

$$
0 \rightarrow \mathcal{O}_{Y}\left(-Y_{1}\right) \rightarrow \mathcal{O}_{Y} \rightarrow \mathcal{O}_{T} \rightarrow 0
$$

to find that $i^{*} i_{*}$ is induced by the functor $\left[\mathcal{O}_{T} \otimes \cdot\right]$. Since $\mathcal{O}_{T}$ is supported in codimension 1 on $Y$, the result follows.

In summation, given $\alpha \in H^{1}\left(\bar{X}, \mathscr{H}_{2}\right)$ and $\beta \in \operatorname{Pic}(\bar{X})$, one computes $\langle\alpha, \beta\rangle$ by the following procedure: (i) Choose a representative $a=\sum_{c}\left[C, h_{C}\right) \in S(\bar{k})$ of $\alpha$. (ii) Use Bertini's theorem to choose a representative $D$ of $\beta$ which meets $a$ properly. (iii) Then

$$
\langle\alpha, \beta\rangle=\prod_{x \in D} \prod_{C} h_{\mathcal{C}}(x)^{t_{x}(C \cdot D)}=\prod_{C} h_{C}(C \cdot D) .
$$

For the sake of completeness, the verification that this description induces a welldefined pairing on $K$-cohomology has been included. Let $\alpha=\sum_{\mathcal{C}}\left[C, h_{C}\right)$ be an element of $S(k)$, so that $\sum_{C} \operatorname{div}\left(h_{C}\right)=0$. Let $D=(g)$ be the divisor of a function. Then, by Weil reciprocity,

$$
\langle\alpha,(g)\rangle=\prod_{C} h_{c}(C \cdot(g))=\prod_{C} h_{C}\left(\left(\left.g\right|_{C}\right)\right)=\left.\prod_{C} g\right|_{c}\left(\left(h_{C}\right)\right)=g\left(\sum_{C}\left(h_{C}\right)\right)=1 .
$$

Therefore, by the moving lemma, $\langle$,$\rangle induces a well-defined pairing between S(\bar{k})$ and $\operatorname{Pic}(\bar{X})$. Further, given any pair of functions $h, g \in \bar{k}(X)^{*}$, another application of Weil reciprocity yields

$$
\langle T\{h, g\}, D\rangle=\langle[(h), g)-[(g), h), D\rangle=\frac{g((h) \cdot D)}{h((g) \cdot D)}=\frac{g\left(\left(\left.h\right|_{D}\right)\right)}{h\left(\left(\left.g\right|_{D}\right)\right)}=1 .
$$

Thus, one actually has a pairing between $H^{1}\left(\bar{X}, \mathscr{K}_{2}\right)$ and $\operatorname{Pic}(\bar{X})$.

Corollary 2.14. Let $\bar{X}$ be any smooth projective surface over an algebraically closed field $k$. Then the pairing (2.11) only depends on the algebraic equivalence class of a divisor, and induces a pairing

$$
H^{1}\left(\bar{X}, \mathscr{X}_{2}\right) \otimes \mathrm{NS}(\bar{X}) \rightarrow \bar{K}^{*} .
$$

Proof. The formula $\prod_{C} h_{C}(C \cdot D)$ visibly only depends on $D$ through the intersection pairing $C \cdot D$, which in turn only depends on the algebraic equivalence class.

Now return to the situation of Lemma 2.10. Let $z$ be a zero cycle of degree zero on a pseudo-rational surface $X$ over the maximal unramified extension $W$ of a local field. Write $z=\sum n_{i} P_{i}$. Next, let $D$ be a $W$-rational $m$-torsion divisor. Assume that the supports of $z$ and $D$ do not meet. Choose a function $f \in W(X)^{*}$ whose divisor is $(f)=m D$. Now write $f(z)=\prod f\left(P_{i}\right)^{n_{i}} \in W^{*} / W^{* m}$. 
Proposition 2.15. For all zero cycles $z$ of degree zero on $X$ and all m-torsion divisors $D$ on $X$ such that $\operatorname{supp}(z) \cap \operatorname{supp}(D)=\emptyset$, one has $\gamma(z) \cup[D] \equiv f(z) \bmod W^{* m}$. Proof. Since $A_{0}(\bar{X})=0$, one can write any zero cycle in the form $z=\sum_{C} \operatorname{div}\left(h_{c}\right)$ for some collection of curves and functions which are defined over $\bar{W}$ and which meet $D$ properly. The isomorphism (Lemma 1.7) between $A_{0}(X)$ and $H^{1}(I, S(\bar{W}))$ identifies $z$ with the 1-cocycle

$$
\sigma \mapsto(1-\sigma) \sum_{C}\left[C, h_{c}\right) .
$$

As written, this cocycle appears to take values in $\coprod_{C} \bar{W}(C)^{*}$. However, since $z$ is defined over $W$, applying the divisor map gives the 0 cocycle in $\coprod_{x \in \mathbb{X}^{2}} \mathbb{Z}$. Thus, it actually takes values in $S(\bar{W})$. The cup product $\gamma(z) \cup[D]$ is, therefore, represented by the 1 -cocycle

$$
\varphi: \sigma \mapsto\left\langle(1-\sigma) \sum_{C}\left[C, h_{C}\right), D\right\rangle=(1-\sigma) \prod_{C} h_{C}(C \cdot D) .
$$

Since $D$ is an $m$-torsion divisor, the latter cocycle takes values in $\mu_{m}$. But every element of $H^{1}\left(I, \mu_{m}\right)=W^{*} / W^{* n}$ is represented by a 1-cocycle of the form

$$
\sigma \mapsto(1-\sigma)^{m} \sqrt{w}
$$

for some $w \in W$. The particular element $w$ which corresponds to the 1-cocycle $\varphi$ is, therefore, a solution to the equation $\sqrt[m]{w}=\prod_{C} h_{C}(C \cdot D)$. Now choose a function $f$ such that $(f)=m D$. Then

$$
\left(\prod_{C} h_{C}(C \cdot D)\right)^{m}=\prod_{C} h_{C}\left(\left(\left.f\right|_{C}\right)\right)=\left.\prod_{C} f\right|_{C}\left(\left(h_{C}\right)\right)=f\left(\sum_{C} \operatorname{div}\left(h_{C}\right)\right)=f(z) .
$$

The result follows.

So far, all these computations apply to an arbitrary pseudo-rational surface over $W$. But many of the statements can be simplified if $X$ has good reduction and if $\operatorname{Pic}(\bar{X})$ has no $p$-torsion. For example, let $N=\operatorname{Pic}(\bar{X}) / P$. Then by Proposition 2.4, the Galois action on the free abelian group $N$ is trivial. Hence, $\left(\operatorname{Pic}(\bar{X}) \otimes \bar{W}^{*}\right)^{i}$ $\approx\left(N \otimes \bar{W}^{*}\right)^{I} \approx N \otimes W^{*}$. Also, the following lemma applies both to $P$ and to its Pontrjagin dual.

Lemma 2.16. Let $M$ be a finite abelian group with trivial I-action. Assume the order of $M$ is relatively prime to $p$. Then $M(1)^{I}=M(1)$ and there is an isomorphism

$$
\delta: H^{1}(I, M(1)) \rightarrow M .
$$

Proof. Let $m$ denote the order of $M$. Since $m$ is prime to $p$, the action of $I$ on $M(1)$ $=M \otimes \mu_{m}$ is trivial. The statement about invariants follows. Furthermore,

$$
H^{1}(I, M(1))=\operatorname{Hom}(I, M(1)) \approx \operatorname{Hom}(\hat{\mathbb{Z}}(1), M(1)) \approx \operatorname{Hom}(\hat{\mathbb{Z}}, M) \approx M .
$$

An explicit realization of this isomorphism is given as follows. There is a unique abelian extension of $W$ of degree $m$, defined by adjoining an $m^{\text {th }}$ root of a uniformizing parameter $\pi$. Fix a primitive $m^{\text {th }}$ root of unity $\zeta$ and a choice of $\sqrt[m]{\pi}$. Let $\tau$ be the generator of the cyclic quotient $\operatorname{Gal}(W(\sqrt[m]{\pi}) / W) \approx \mathbb{Z} / m$ of $I$ determined 
by $\tau(\sqrt[m]{\pi})=\zeta \sqrt[m]{\pi}$. Then every element $\lambda \in H^{1}(I, M(1))$ is completely determined by $\lambda(\tau)$. Now write $\lambda(\tau)=x \otimes \zeta$ for some $x \in M$. Then the isomorphism is given by $\delta(\lambda)=x$.

Finally, one can compute the group of zero cycles on a pseudo-rational surface $X$ with good reduction over the maximal unramified extension $W$.

Proposition 2.17. Let $X$ be a pseudo-rational surface with good reduction over $W$. If the residue characteristic of $W$ is relatively prime to the exponent $m$ of the torsion group $P$, then $A_{0}(X)=0$.

Proof. Let $\mathscr{X}$ be a smooth proper model of $X$ over the ring of integers $\mathcal{O}_{W}$ in $W$. By Lemma 2.10, it is enough to show that the image of $\gamma$ is zero. This image can be computed using Proposition 2.15. Choose a function $f \in W(X)^{*}$ whose divisor is $(f)=m D$ on $\mathscr{X}$. Let $z \in X$ be a closed point, and let $z_{0}$ be its specialization. If $f\left(z_{0}\right) \neq 0$ or $\infty$, then $f(z) \in \mathcal{O}_{W}^{*}$ and, since $\operatorname{gcd}(m, p)=1$, it must be an $m^{\text {th }}$ power. So, it is enough to show that if $f\left(z_{0}\right)=0$ or $\infty$, then $v(f(z))$ is a multiple of $m$, where $v$ is the valuation on $W$. Work in the local ring of $z_{0}$ on $\mathscr{X}$. Since this is a regular local ring and $(f)=m D$, one can write $f=w^{m} u$ locally, where $u$ is a unit. But then $v(f(z))$ $=m v(w(z))$.

Corollary 2.18. With the above hypotheses, $H^{2}(I, R(\bar{W})) \approx P^{D}$.

Proof. Since $X$ has good reduction, Hensel's Lemma supplies a $W$-rational point on $X$. Taking the Galois cohomology of sequence (1.2), one sees that $H^{2}(I, S(\bar{W}))$ $=H^{1}\left(I,(\amalg \mathbb{Z})^{0}\right)=0$. Use the previous proposition to take the cohomology of sequence (1.3), and apply Lemma 2.6 and Lemma 2.16 to obtain

$$
H^{2}(I, R(\bar{W})) \approx H^{1}\left(I, H^{1}\left(\bar{X}, \mathscr{K}_{2}\right)\right) \approx H^{1}\left(I, P^{D}(1)\right) \approx P^{D} .
$$

\section{Local fields}

It will be useful to standardize notation while studying pseudo-rational surfaces over a $p$-adic field $L$. Let $A$ be the ring of integers, $\pi$ a uniformizing parameter, and $F=\Lambda / \pi$ the finite residue field of $L$. Let $W$ be the maximal unramified extension of $L$. Denote the Galois groups by: $G=\operatorname{Gal}(\bar{L} / L), I=\operatorname{Gal}(\bar{L} / W)$, and $\Gamma=G / I$ $\approx \operatorname{Gal}(W / L) \approx \operatorname{Gal}(\bar{F} / F) \approx \hat{\mathbb{Z}}$. Let $X$ be a pseudo-rational surface over $L$. Throughout this section, it will be assumed that the characteristic of $F$ is relatively prime to the torsion subgroup $P$ of $\operatorname{Pic}(\bar{X})$. Finally, let $N=\operatorname{Num}(\bar{X})=\operatorname{Pic}(\bar{X}) / P$. Some preliminary computations of Galois cohomology will prove useful.

Lemma 3.1. Let $X$ be a pseudo-rational surface defined over a p-adic field $L$. Then

$$
H^{1}(G, R(\bar{L}))=H^{1}\left(\Gamma, H^{0}(I, R(\bar{L}))\right) .
$$

Proof. Use Lemma 2.7 and the Serre spectral sequence.

Lemma 3.2. Let $X$ be a pseudo-rational surface defined over a p-adic field $L$. Then

$$
H^{1}(\Gamma, R(W))=0 .
$$

Proof. Take the cohomology of the first exact sequence of Corollary 2.9. The result follows from Colliot-Thélène's result [7] that $H^{1}(\Gamma, Q(W))=0$ and from the fact that $\Gamma \approx \hat{Z}$ has cohomological dimension one $[33,34]$. 
Lemma 3.3. Let $X$ be a pseudo-rational surface defined over a p-adic field $L$. The isomorphism $\delta: H^{1}(I, P(1)) \rightarrow P$ is an isomorphism of $\Gamma$-modules.

Proof. Since the order of $P$ is prime to the residue characteristic, $I$ acts tamely. But the tame inertia group is isomorphic to $\prod_{l \neq p} \mathbb{Z}_{l}(1)$ as a $\Gamma$-module. The result follows.

The remainder of this section applies to surfaces with "very good reduction." It is not enough to assume that a smooth, proper model exists over the ring of integers. Problems can arise (for example, when trying to lift line bundles) because the dimension of the Zariski cohomology of the structure sheaf need not be constant in families.

Definition 3.4. A pseudo-rational surface $X$ over $L$ will be said to have very good reduction if there exists a smooth, proper model $\mathscr{X} \rightarrow \operatorname{Spec}(\Lambda)$ whose closed fibre $Y \rightarrow \operatorname{Spec}(F)$ is also pseudo-rational.

Very good reduction is a reasonably well-behaved property of pseudo-rational surfaces. It is preserved under unramified base extension, since smoothness and properness are and since the definition of pseudo-rational only depends on the nature of the surface over the algebraic closure. Also, if $X$ is a pseudo-rational surface over a number field, then it has very good reduction at all but finitely many primes. The main point is to use the semicontinuity theorem to ensure that $p_{g}$ and $q$ remain zero at almost all primes.

Lemma 3.5. Let $X$ be a pseudo-rational surface over $L$ with very good reduction. Then $X_{W}$ also has very good reduction to $\vec{Y}$, and there are canonical isomorphisms

$$
\operatorname{Pic}(\bar{X}) \leftarrow \operatorname{Pic}\left(X_{W}\right) \leftarrow \operatorname{Pic}(\tilde{X}) \rightarrow \operatorname{Pic}(\bar{Y}) \text {. }
$$

Proof. The first map is an isomorphism because, by Proposition 2.4, $W$ splits $X$. Now let $\widetilde{X} \rightarrow \operatorname{Spec}\left(\mathcal{O}_{W}\right)$ be the scheme obtained from $\mathscr{X} \rightarrow \operatorname{Spec}(A)$ by base extension to the ring of integers in $W$; this is a smooth, proper model of $X_{W}$. Divisors on $\widetilde{X}$ are the same as those on $X_{W}$ except for the closed fibre, which is the divisor of the "constant" function $\pi$. The second arrow is, therefore, also an isomorphism. The closed fibre of $\widehat{X}$ is canonically isomorphic to $\bar{Y}=Y \otimes_{F} \bar{F}$, and its normal bundle in $\widetilde{\mathscr{X}}$ is isomorphic to $\mathcal{O}_{\overline{\mathrm{Y}}}$. Since the reduction is very good, the cohomology of the normal bundle vanishes; this ensures the uniqueness and existence of vector bundles on the formal completion of $\widetilde{X}$ along $\bar{Y}$ which lift bundles on $\bar{Y}$. That the final arrow is an isomorphism now follows from Corollary 1 of Proposition 3 of [21].

Theorem 3.6. Let $X$ be a pseudo-rational surface over a p-adic field L. If $X$ has very good reduction over $L$, then the map $A_{0}(X) \rightarrow H^{1}\left(G, H^{1}\left(\bar{X}, \mathscr{K}_{2}\right)\right)$ is injective.

Proof. Since both $W$ and $F$ have cohomological dimension 1, the Serre spectral sequence and Lemma 3.3 give isomorphisms

$$
H^{2}(G, P(1)) \approx H^{1}\left(\Gamma, H^{1}(I, P(1))\right) \approx H^{1}(\Gamma, P) .
$$

Using Lemmas 3.2 and 3.3, the cohomology of the second sequence of Corollary 2.9 yields an exact sequence

$$
0 \rightarrow H^{1}\left(\Gamma, R(\bar{L})^{I}\right) \rightarrow H^{1}(\Gamma, P) \stackrel{\star}{\longrightarrow} H^{2}(\Gamma, R(W)) .
$$


Now take an arbitrary element $\varphi \in H^{1}(\Gamma, P)$. By Lemma 3.1, it is enough to verify the statement:

$$
\text { if } \alpha(\varphi)=0 \text {, then } \varphi \text { is in the image of }\left(\operatorname{Pic}(\bar{X}) \otimes \bar{L}^{*}\right)^{G} \text {. }
$$

Observe that the usual tame symbol $K_{2}(W(X)) \rightarrow \bar{F}(Y)^{*}$ naturally induces a homomorphism $T: R(W) \rightarrow \bar{F}(Y)^{*} / \bar{F}^{*}$. The map on Galois cohomology will also be denoted by $T$. It now suffices to verify statement (3.7) with $\alpha$ replaced by the composite $T \alpha$.

Fortunately, this composite can be computed explicitly. The strategy is as follows. Since $\alpha$ is a connecting homomorphism for the $\Gamma$-cohomology of the exact sequence (2.9)

$$
0 \rightarrow R(W) \rightarrow R(\bar{L})^{I} \stackrel{\beta}{\longrightarrow} P \rightarrow 0,
$$

$\alpha(\varphi)$ will be computed by first lifting the 1 -cocycle $\varphi$ on $\Gamma$ with values in $P$ along $\beta$ to a 1-cochain $\psi$ with values in $R(\bar{L})^{I}$. The coboundary of $\psi$ produces a 2-cocycle with values in $R(W)$. In order to describe the lifting, one must recall that $\beta$ is, itself, a connecting homomorphism for the $I$-cohomology of the exact sequence (1.8)

$$
0 \rightarrow P(1) \rightarrow Q(\bar{L}) \rightarrow R(\bar{L}) \rightarrow 0 .
$$

After applying the tame symbol, the assumption that $T \alpha(\varphi)$ is a coboundary will be used to produce a Galois invariant element of $\operatorname{Pic}(\bar{X}) \otimes \bar{L}^{*}$ which maps onto $\varphi$.

This is how to lift elements along $\beta$ : Let $D$ be a $W$-rational $m$-torsion divisor. Choose $f \in W(X)^{*}$ with divisor $(f)=m D$. Consider the element $\{f, \sqrt[m]{\pi}\}$ $\in K_{2}(\bar{L}(X))$. Let $\tau$ be the element of $I$ described in Lemma 2.16. One has

$$
(\tau-1)\{f, \sqrt[m]{\pi}\}=\{f, \zeta \sqrt[m]{\pi}\}\{f, \sqrt[m]{\pi}\}^{-1}=\{f, \zeta\} .
$$

Since $\{f, \zeta\} \in H^{0}\left(\bar{X}, \mathscr{K}_{2}\right)$, this computation shows that $\{f, \sqrt[m]{\pi}\}$ represents an I-invariant element of $R(\bar{L})$. Write $\lambda=\beta(\{f, \sqrt[m]{\pi}\})$, viewed in $H^{1}(I, P(1)) \approx P$. It follows from Lemma 1.6 that $\lambda(\tau)=\{f, \zeta\}$ corresponds to $[D] \otimes \zeta$. Using Lemma 3.3 to identify $P$ with $H^{1}(I, P(1))$, one sees that $\beta(\{f, \sqrt[m]{\pi}\})=[D]$.

Let $\varphi(\gamma)=\left[D_{\gamma}\right]$ be a 1-cocycle on $\Gamma$ with values in $P$. Choose functions $f_{y} \in W(X)^{*}$ with divisors $\left(f_{y}\right)=m D_{\gamma}$. Then $\varphi$ can be lifted to the 1-cochain $\psi$ on $\Gamma$ with values in $R(\bar{L})^{I}$ defined by the formula

$$
\psi: \gamma \mapsto\left\{f_{\gamma}, \sqrt[m]{\pi}\right\} .
$$

Thus, $\alpha(\varphi)$ is the 2-cocycle on $\Gamma$ with values in $R(W)$ defined by

$$
\alpha(\varphi)(\gamma, \delta)=\partial \psi(\gamma, \delta)=\frac{\gamma\left\{f_{\delta}, \sqrt[m]{\pi}\right\}\left\{f_{\gamma,}^{m} \sqrt{\pi}\right\}}{\left\{f_{\gamma \delta}, \sqrt[m]{\pi}\right\}} \equiv\left\{\frac{\gamma}{f_{\delta} f_{\gamma}}, \sqrt[m]{\pi}\right\} \bmod H^{0}\left(X_{W}, \mathscr{K}_{2}\right) .
$$

However, since $\varphi$ is a 1-cocycle with values in $P$, there are functions $g_{y, \delta} \in W(X)^{*}$ with divisors

$$
\left(g_{\gamma, \delta}\right)={ }^{\gamma} D_{\delta}+D_{\gamma}-D_{\gamma \delta}
$$

Hence,

$$
\left(g_{\gamma, \delta}^{m}\right)=\left(\frac{\gamma_{\delta} f_{\gamma}}{f_{\gamma \delta}}\right)
$$


Therefore, the functions whose divisors appear on either side of this equality must differ only by a constant.

A delicate point intervenes. By Lemma 3.5, the torsion divisor classes $\left[D_{\gamma}\right]$ may be represented by horizontal divisors on $\widetilde{\mathscr{X}}$, and the identities $\left(f_{\gamma}\right)=m D_{y}$ and $\left(g_{\gamma, \delta}\right)$ $={ }^{\gamma} D_{\delta}+D_{\gamma}-D_{\gamma \delta}$ may be assumed to hold in $\operatorname{Div}(\widetilde{\mathscr{X}})$. With these assumptions, the function ${ }^{\gamma} f_{\delta} f_{\gamma} / f_{\gamma, \delta} g_{\gamma, \delta}^{m}$ is actually a constant in $\mathcal{O}_{W}^{*}$. Since $\mathcal{O}_{W}^{*}$ is $m$-divisible, the choice of $g_{y, \delta}$ can be modified to ensure that this constant always equals 1 . Composing with the tame symbol, one has

$$
T \alpha(\varphi)(\gamma, \delta)=T\left(\left\{g_{\gamma, \delta}, \pi\right\}\right) \equiv\left(g_{\gamma, \delta}\right) \bmod \pi .
$$

The next task is to determine when this 2-cocycle with values in $\bar{F}(Y)^{*} / \bar{F}^{*}$ is cohomologically trivial, and then to use that information to produce a Galois invariant element of $\operatorname{Pic}(\bar{X}) \otimes \bar{L}^{*}$. Since the cohomology of $\mathbb{G}_{m}$ over a finite field vanishes, there is an isomorphism

$$
H^{2}\left(\Gamma, \bar{F}(Y)^{*}\right) \approx H^{2}\left(\Gamma, \bar{F}(Y)^{*} / \bar{F}^{*}\right) .
$$

The cocycle $T \alpha(\varphi)$, viewed with values in $\bar{F}(Y)^{*}$, is given by the above formula with the divisor $(g)$ replaced by the function $g$. This cocycle only represents a trivial cohomology class if it is a coboundary. More precisely, $T \alpha(\varphi)=0$ if and only if there exist functions $b_{y} \in \bar{F}(Y)^{*}$ which satisfy the equations

$$
\frac{{ }^{\gamma} b_{\delta} b_{\gamma}}{b_{\gamma \delta}} \equiv g_{y, \delta} \bmod \pi \text {. }
$$

Let $D_{\gamma}^{\prime}$ denote the pullback of $D_{\gamma}$ to the closed fibre $\bar{Y}$. Define $E_{\gamma}=D_{\gamma}^{\prime}-\left(b_{\gamma}\right)$. The assignment $\gamma \mapsto m E_{\gamma}$ is a 1-cocycle on $\Gamma$ with values in $\operatorname{Div}(\bar{Y})$. For,

$$
m\left(\frac{{ }^{\gamma} b_{\delta} b_{\gamma}}{b_{\gamma \delta}}\right)=m\left(g_{\gamma, \delta} \bmod \pi\right)=\left(\frac{{ }^{\gamma} f_{\delta} f_{\gamma}}{f_{\gamma \delta}} \bmod \pi\right)=m\left({ }^{\gamma} D_{\delta}^{\prime}+D_{\gamma}^{\prime}-D_{\gamma \delta}^{\prime}\right) .
$$

But $\operatorname{Div}(\bar{Y})$ is torsion free, so that $\gamma \mapsto E_{\gamma}$ must also be a 1-cocycle. Now the vanishing of the first cohomology group of this permutation module implies that there exists a divisor $C \in \operatorname{Div}(\bar{Y})$ such that $E_{\gamma}=(\gamma-1) C$ for all $\gamma \in \Gamma$.

Use the isomorphism (Lemma 3.5) between $\operatorname{Pic}(\bar{X})$ and $\operatorname{Pic}(\bar{Y})$ to interpret the divisor class $[C]$ as an element of the former group. Consider the element $[C] \otimes \pi \in \operatorname{Pic}(\bar{X}) \otimes \bar{L}^{*}$. By construction, this element is $I$-invariant. Moreover,

$$
(\gamma-1)([C] \otimes \pi)=(\gamma-1)[C] \otimes \pi=\left[E_{\gamma}\right] \otimes \pi=\left[D_{\gamma}\right] \otimes \pi-\left(b_{\gamma}\right) \otimes \pi=\left[D_{\gamma}\right] \otimes \pi .
$$

But $\left[D_{\gamma}\right]$ is a torsion divisor class and $\bar{L}^{*}$ is divisible, so $\left[D_{y}\right] \otimes \pi$ is trivial. Therefore, $[C] \otimes \pi$ is Galois invariant.

To complete the proof, it only remains to show that $[C] \otimes \pi$ maps onto $\varphi$ under the composite

$$
\begin{aligned}
\left(\operatorname{Pic}(\bar{X}) \otimes \bar{L}^{*}\right)^{G} & \rightarrow H^{1}\left(\bar{X}, \mathscr{K}_{2}\right)^{G} \rightarrow H^{1}(G, R(\bar{L})) \\
& \rightarrow H^{2}(G, P(1)) \approx H^{1}\left(\Gamma, H^{1}(I, P(1))\right) .
\end{aligned}
$$

Represent the divisor class $[C] \in \operatorname{Pic}(\bar{X})=\operatorname{Pic}\left(X_{W}\right)$ by a divisor $\Delta=\sum n_{i} \Delta_{i} \in \operatorname{Div}\left(X_{W}\right)$. The image of $[C] \otimes \pi$ in $H^{1}\left(\bar{X}, \mathscr{K}_{2}\right)$ is represented by the class of

$$
[\Delta, \pi)=\sum\left[\Delta_{i}, \pi^{n_{i}}\right) \in S(\bar{L}) \subset \coprod_{x \in \bar{X}^{1}} \bar{L}(x)^{*} .
$$


The image of $[C] \otimes \pi$ in $H^{1}(G, R(\bar{L}))$ is, therefore, represented by the 1-cocycle $\sigma \mapsto(\sigma-1)[\Delta, \pi)$. Of course, the latter element must be appropriately interpreted as an element of $R(\bar{L})$. One may again assume that $\Delta$ is actually a horizontal divisor on $\widetilde{X}$. Then $\Delta$ is $I$-invariant and for all $\gamma \in \Gamma$ one has

$$
(\gamma-1)[\Delta]=(\gamma-1)[C]=\left[E_{\gamma}\right]=\left[D_{\gamma}\right] .
$$

Here the intermediate steps should be thought of as making sense after pulling back to the geometric special fibre, even though the extreme classes are represented by divisors on the model. Since the isomorphisms of Picard groups are given by pullbacks, there is no danger here. Thus, one can find functions $\varrho_{\gamma}$ with divisors $\left(\varrho_{\gamma}\right)=(\gamma-1) \Delta-D_{\gamma}$, where the equality once again holds in $\operatorname{Div}(\tilde{\mathscr{X}})$. Further, one has

$$
\left(f_{\gamma} \varrho_{\gamma}^{m}\right)=m D_{\gamma}+m\left((\gamma-1) \Delta-D_{\gamma}\right)=m(\gamma-1) \Delta .
$$

Thus, the element $\left\{f_{\gamma} e_{\gamma}^{m}, \sqrt[m]{\pi}\right\} \in K_{2}(\bar{L})$ represents $(\gamma-1)[\Delta, \pi)$. Therefore, the image of $[C] \otimes \pi$ in $H^{1}(G, R(\bar{L})) \approx H^{1}\left(\Gamma, R(\bar{L})^{T}\right)$ is given by the 1-cocycle

$$
\gamma \mapsto\left\{f_{\gamma} \varrho_{\gamma}^{m}, \sqrt[m]{\pi}\right\} \bmod H^{0}\left(\bar{X}, \mathscr{K}_{2}\right)^{I} .
$$

To finish the computation of the image of $[C] \otimes \pi$ along the composite, notice that

$$
\left\{f_{\gamma} \varrho_{\gamma}^{m}, \sqrt[m]{\pi}\right\}=\left\{f_{\gamma}, \sqrt[m]{\pi}\right\}\left\{\varrho_{\psi}, \pi\right\}
$$

Since $\left\{\varrho_{y}, \pi\right\}$ is actually an element of $K_{2}(W(X))$, it dies in $H^{1}(I, P(1))$. Thus, the image of $[C] \otimes \pi$ in $H^{1}\left(\Gamma, H^{1}(I, P(1))\right)$ is the same as the image of the cocycle $\gamma \mapsto\left\{f_{y}, \sqrt[m]{\pi}\right\}$. But that is precisely the cocycle which was shown earlier to represent $\varphi$. The theorem follows.

The proof of the theorem actually shows something stronger than the stated injectivity. This stronger result will play a role in the proof of the finiteness theorem for zero cycles on pseudo-rational surfaces over number fields.

Corollary 3.8. Let $X$ be a pseudo-rational surface with very good reduction over a p-adic field $L$. Then the natural map $\left(\operatorname{Pic}(\bar{X}) \otimes \bar{L}^{*}\right)^{G} \rightarrow H^{1}(G, R(\bar{L}))$ is a surjection.

Proof. This is a restatement of (3.7).

Lemma 3.9. Let $X$ be a pseudo-rational surface with very good reduction over a p-adic field $L$. Then the natural map $H^{1}\left(G, \operatorname{Pic}(\bar{X}) \otimes \bar{L}^{*}\right) \rightarrow H^{1}\left(G, H^{1}\left(\bar{X}, \mathscr{K}_{2}\right)\right)$ is injective. Consequently, the natural map $H^{1}\left(\bar{X}, \mathscr{K}_{2}\right)^{G} \rightarrow P^{D}(1)^{G}$ is surjective.

Proof. The second statement follows from the first and from the cohomology exact sequence of (1.5). So, write $U=\operatorname{Pic}(\bar{X}) \otimes \bar{L}^{*}$. It suffices to show that every element of $H^{1}(G, U)$ is detected by the pairing (2.11)

$$
H^{1}\left(\bar{X}, \mathscr{K}_{2}\right) \otimes \operatorname{Pic}(\bar{X}) \rightarrow \bar{L}^{*} .
$$

By local duality, $H^{1}(G, U)$ is dual to $H^{1}(G, N)$. Since $U$ is divisible, $H^{1}(G, U)$ pairs trivially with the Galois cohomology of the torsion subgroup of $\operatorname{Pic}(\bar{X})$. Therefore, it is enough to show that the natural map $H^{1}(G, \operatorname{Pic}(\bar{X})) \rightarrow H^{1}(G, N)$ is surjective. Since the maximal unramified extension is a splitting field, the Serre spectral 
sequence yields an isomorphism $H^{1}(G, N) \approx H^{1}(\Gamma, N)$. The result now follows from the exact commutative diagram

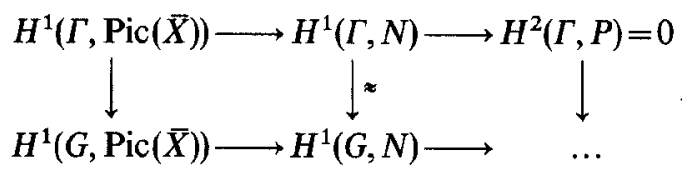

Proposition 3.10. Let $X$ be a pseudo-rational surface with very good reduction over a p-adic field $L$. Then $\gamma: A_{0}(X) \rightarrow H^{1}\left(G, P^{D}(1)\right)$ is injective.

Proof. As before, $W$ splits $X$. Using Lemma 2.10, one sees that $\operatorname{Ker}(\gamma)$ must be contained in $\operatorname{Ker}\left(A_{0}(X) \rightarrow A_{0}\left(X_{W}\right)\right)$. By Lemma 1.7, the Serre spectral sequence for $S(\bar{L})$ leads to an isomorphism between the latter kernel and $H^{1}(\Gamma, S(W))$. The following analogue of Proposition 1.9 will prove useful:

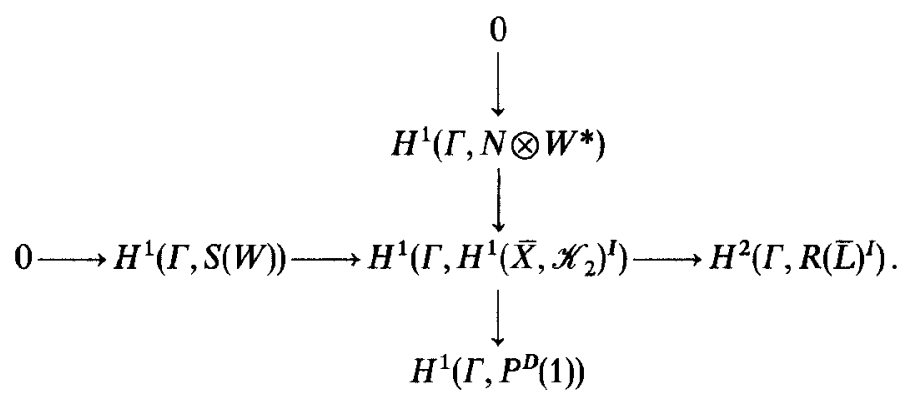

Here the horizontal row is the $\Gamma$-cohomology of the exact sequence

$$
0 \rightarrow R(\bar{L})^{I} \rightarrow S(W) \rightarrow H^{1}\left(\bar{X}, \mathscr{K}_{2}\right)^{I} \rightarrow 0,
$$

which exists by Lemma 2.7. Injectivity in the horizontal row follows from functoriality of the Serre spectral sequence and from Theorem 3.6. The vertical column above is the $\Gamma$-cohomology of the exact sequence

$$
0 \rightarrow N \otimes W^{*} \rightarrow H^{1}\left(\bar{X}, \mathscr{K}_{2}\right)^{I} \rightarrow P^{D}(1) \rightarrow 0,
$$

which exists by Lemma 2.6. Injectivity in the vertical column is a consequence of the functoriality of the Serre spectral sequence and of Lemma 3.9.

Define $A$ to be the kernel of the composite

$$
H^{1}\left(\Gamma, \operatorname{Pic}\left(X_{W}\right) \otimes W^{*}\right) \rightarrow H^{1}\left(\Gamma, H^{1}\left(X_{W}, \mathscr{K}_{2}\right)\right) \rightarrow H^{2}(\Gamma, R(W)) .
$$

By Corollary 2.9 , there is an exact commutative diagram

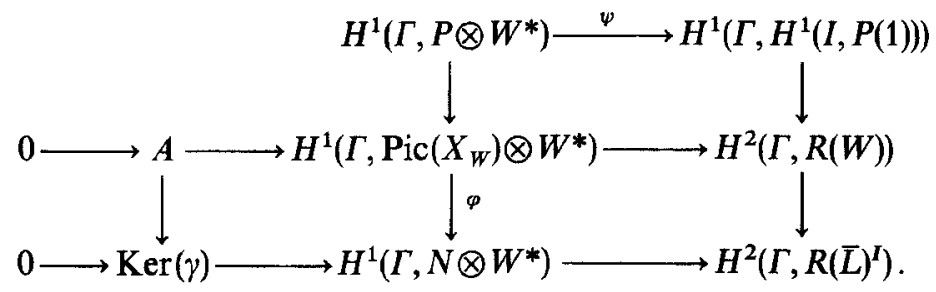

It is straightforward to check that $\psi$ is an isomorphism, compatible with the identifications

$$
v: P \otimes W^{*} \stackrel{\star}{\longrightarrow} P \text { and } \delta: H^{1}(I, P(1)) \stackrel{\star}{\longrightarrow} P .
$$


Since $c d(\Gamma)=1$, the map $\varphi$ is surjective. Now a diagram chase shows that $\operatorname{Ker}(\gamma)$ is a quotient of $A$. Therefore, it is enough to show that $A=0$.

Let $\mathcal{O}_{W}$ be the ring of integers in $W$. Since the residue characteristic is prime to the exponent $m$ of $P, \mathcal{O}_{W}^{*}$ is $m$-divisible. Since the Picard group only contains $m$-torsion, it follows that $\operatorname{Pic}\left(X_{W}\right) \otimes \mathcal{O}_{W}^{*} \approx N \otimes \mathcal{O}_{W}^{*}$. Because $W / L$ is unramified, one may use Proposition 47 of [34] to conclude that both $\mathcal{O}_{W}^{*}$ and $N \otimes \mathcal{O}_{W}^{*}$ are cohomologically trivial. Thus, there are isomorphisms

$$
v: H^{1}\left(\Gamma, \operatorname{Pic}\left(X_{W}\right) \otimes W^{*}\right) \stackrel{*}{\longrightarrow} H^{1}\left(\Gamma, \operatorname{Pic}\left(X_{W}\right) \stackrel{\bullet}{\longrightarrow} H^{1}(\Gamma, \operatorname{Pic}(\bar{Y})),\right.
$$

where the latter isomorphism again uses Lemma 3.5. As in Bloch's proof, [3, Theorem 7.12], of the analogous result for rational surfaces, one can work on a model $\widetilde{X}$ of $X_{W}$ over $\mathscr{O}_{W}$ to produce a commutative diagram

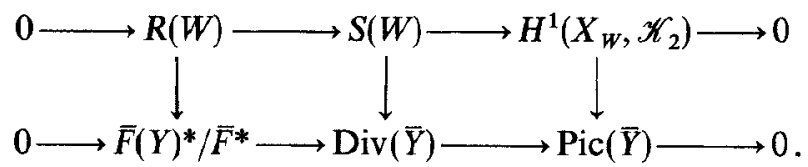

Here the first vertical arrow is induced by the tame symbol. The middle vertical arrow can be described as follows. Given $\sum_{C}\left[C, h_{C}\right) \in S(W)$, take the closure of each curve $C$ in $\tilde{\mathscr{X}}$. These are arithmetic surfaces; taking the divisor of $h_{C}$ gives a sum of curves with multiplicities on $\overline{\mathscr{X}}$. Summing over all $C$ and using the fact that the part of the divisor supported on the generic fibre cancels, one gets a sum of curves with multiplicities supported on the closed fibre; i.e., a divisor. It is clear from this description that the induced map $\operatorname{Pic}\left(X_{W}\right) \otimes W^{*} \rightarrow H^{1}\left(X_{W}, \mathscr{K}_{2}\right) \rightarrow \operatorname{Pic}(\bar{Y})$ is "specialization times valuation." Now taking cohomology gives a commutative diagram

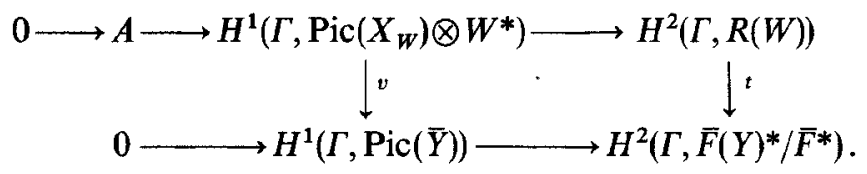

The result follows.

Theorem 3.11. Let $X$ be a pseudo-rational surface with very good reduction over a $p$-adic field $L$. Assume that $p$ is prime to the order of $P$. Then

$$
A_{0}(X) \approx H^{1}\left(\Gamma, P^{D}(1)\right) \approx A_{0}(Y) .
$$

Proof. The second isomorphism is Proposition 2.1. Let $X \rightarrow \operatorname{Spec}(\Lambda)$ be a smooth proper model of $X$ over the ring of integers $A$. By Hensel's Lemma, the specialization map from zero cycles of degree 0 on $X$ to zero cycles of degree 0 on the reduction $Y$ is surjective. So, $A_{0}(X)$ must be at least this large. It is now enough to show that the image of $\gamma$ actually lands in the unramified subgroup $H^{1}\left(\Gamma, P^{D}(1)\right)$. But there is a commutative square

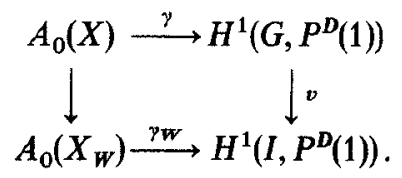

The kernel of $v$ can naturally be identified with $H^{1}\left(\Gamma, P^{D}(1)\right)$. The result follows from the injectivity of $\gamma$ (Proposition 3.10) and the vanishing of $A_{0}\left(X_{W}\right)$ (Proposition 2.17). 


\section{Number fields}

In this section, $X$ denotes a pseudo-rational surface over a number field $k$. Since it will become necessary to deal with Galois cohomology over various fields simultaneously, write $H^{1}(k, A)$ instead of $H^{1}(\operatorname{Gal}(\bar{k} / k), A)$. Define

$$
\begin{gathered}
B_{0}(X)=\operatorname{Ker}\left(A_{0}(X) \rightarrow H^{1}\left(k, P^{D}(1)\right)\right), \\
C_{0}(X)=\operatorname{Ker}\left(A_{0}(X) \rightarrow H^{1}\left(k, H^{1}\left(\bar{X}\left(\bar{X}, \mathscr{K}_{2}\right)\right)\right) .\right.
\end{gathered}
$$

Lemma 4.1. If $X$ is a pseudo-rational surface over a number field $k$, then $C_{0}(X)$ is finite.

Proof. Choose a finite Galois invariant set of curves which generates $\operatorname{Pic}(\bar{X})$. Let $D$ be the free abelian group of divisors generated by this set of curves. Define $V$ to be the torus which is the kernel of the natural map $D \otimes \bar{k}^{*} \rightarrow \operatorname{Pic}(\bar{X}) \otimes k^{*}$. There is a commutative diagram

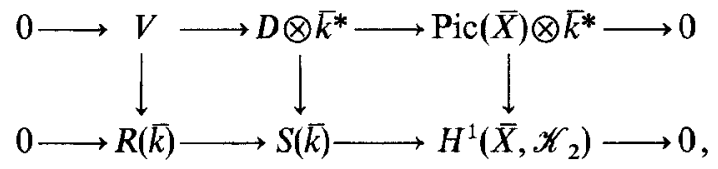

in which all the vertical arrows are inclusions. Since $D$ is a permutation module, the Galois cohomology group $H^{1}\left(k, D \otimes \bar{k}^{*}\right)$ vanishes. Using Lemma 1.7 , one obtains an exact diagram of cohomology groups

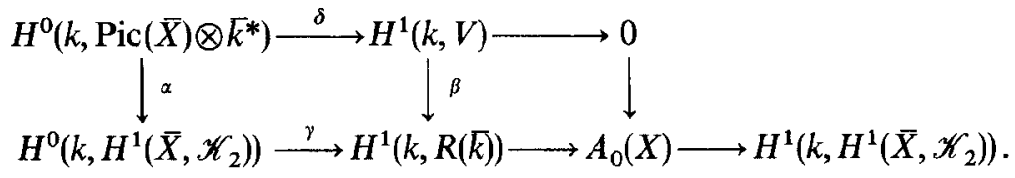

To prove the lemma, one must show that $\operatorname{Coker}(\gamma)$ is finite. Since $\alpha$ has finite cokernel and $\delta$ is surjective, it is enough to show that $\operatorname{Coker}(\beta)$ is finite.

Notice first that one has the same diagram if $k$ is replaced by a local field whose residue characteristic is prime to the order of $P$, over which $X$ has very good reduction. By Corollary 3.8, the local version of the composite $\gamma \alpha$ is surjective. Hence the local version of $\beta$ is also surjective. Moreover, there is a (non-exact) commutative diagram relating the local and global situations.

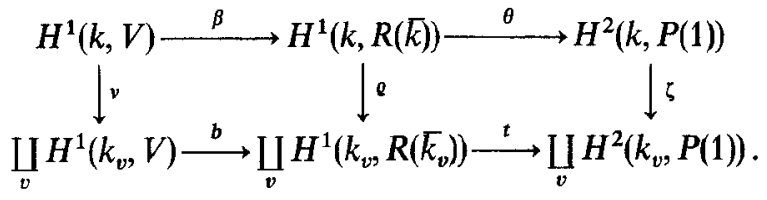

Both $\theta$ and $t$ are injective by Proposition 1.9. Tate [37] has shown that $\zeta$ has finite kernel. So, the map $\varrho$ also has finite kernel. Thus, in order to verify the finiteness of Coker $(\beta)$, it is enough to show that both $v$ and $b$ have finite cokernels. For $\psi$, this follows from Tate [38]. Since $b$ is the sum of the local versions of $\beta$, its cokernel comes from finitely many primes: archimedean, bad reduction, and primes dividing the order of $P$. Therefore, $\operatorname{Coker}(\beta)$ must also be finite.

Proposition 4.2. If $X$ is a pseudo-rational surface over a number field $k$, then $B_{0}(X)$ is finite. 
Proof. By the lemma, it is enough to show that $D_{0}(X)=B_{0}(X) / C_{0}(X)$ is finite. Let $U$ denote the torus $\operatorname{Pic}(\bar{X}) \otimes k^{*}$. Let $\delta: P^{D}(1)^{G} \rightarrow H^{1}(G, U)$ be the connecting homomorphism resulting from the Galois cohomology of the exact sequence

$$
0 \rightarrow U \rightarrow H^{1}\left(\bar{X}, \mathscr{K}_{2}\right) \rightarrow P^{D}(1) \rightarrow 0 .
$$

By comparing the global groups with the local groups, one gets a commutative diagram which defines $\amalg(D)$ and $\Psi^{\prime}(k, U)$ :

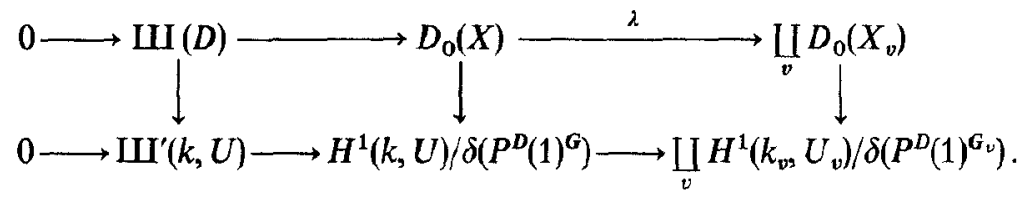

Since the vertical arrows are injective, it is enough to show that both the image of $\lambda$ and $\Psi^{\prime}(k, U)$ are finite. Since $X$ has very good reduction at almost all $v$, Proposition 3.10 shows that the image of $\lambda$ sits inside a finite direct sum. It remains, therefore, to show that $\Psi^{\prime}(k, U)$ is always finite.

Consider the exact commutative diagram

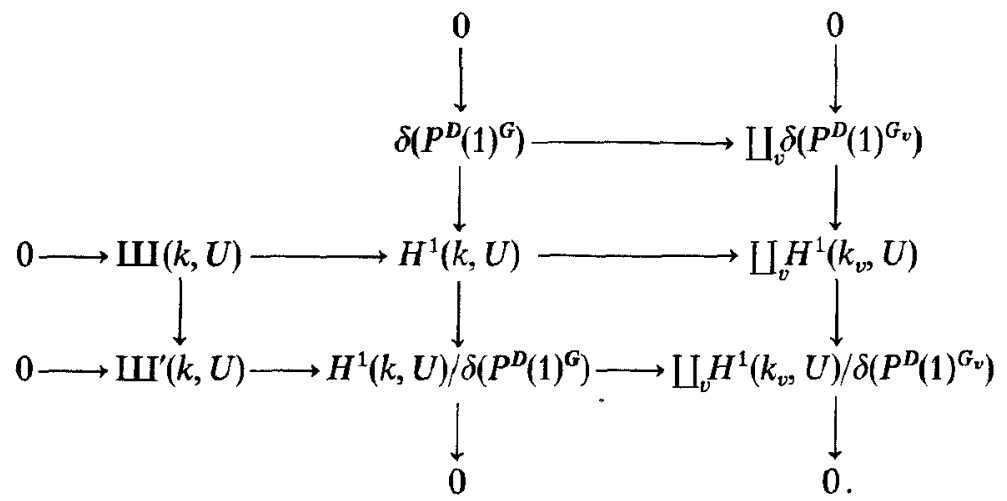

Since $\amalg(k, U)$ is finite [38], a straightforward diagram chase shows that it is enough to verify that $\delta\left(P^{D}(1)^{G_{v}}\right)=0$ for almost all $v$. But this follows immediately from Lemma 3.9.

The argument above is essentially standard. It requires, however, that the local invariants be almost always zero. The same argument cannot be used to prove the finiteness of $A_{0}(X)$, because Theorem 3.11 shows that the latter is almost never zero. Nevertheless, a variation of this argument, based on the proof of the weak Mordell-Weil Theorem, will work.

Theorem 4.3. Let $X$ be a pseudo-rational surface over a number field $k$. Then $A_{0}(X)$ is a finite group.

Proof. By the previous proposition, it suffices to show that the image of

$$
\gamma: A_{0}(X) \rightarrow H^{1}\left(k, P^{D}(1)\right)
$$

is finite. Let $S$ be a finite set of primes including the infinite primes, the primes dividing the order of $P$, and the primes of bad reduction. For each $p \notin S$, the composite $A_{0}(X) \rightarrow H^{1}\left(k, P^{D}(1)\right) \rightarrow H^{1}\left(k_{p}, P^{D}(1)\right)$ factors through $A_{0}\left(X_{k_{p}}\right)$. But by 
Proposition 2.17, one has $A_{0}\left(X_{k^{p n}}\right)=0$. Thus, the image of $\gamma$ is contained in the kernel of

$$
H^{1}\left(k, P^{D}(1)\right) \rightarrow \coprod_{p \notin S} H^{1}\left(k_{p}, P^{D}(1)\right) \rightarrow \coprod_{p \notin S} H^{1}\left(k_{p}^{\mathrm{un}}, P_{D}(1)\right) .
$$

Therefore, the image of $\gamma$ is contained in the part of the cohomology group generated by cocycles which are unramified outside the finite set of primes in $S$. To see that this is a finite group, one can first make a finite field extension and assume that the action of the Galois group $G=\mathrm{Gal}(k / k)$ on the finite module $P^{D}(1)$ is trivial. Then the first cohomology group can be identified with $\operatorname{Hom}\left(G, P^{D}(1)\right)$. Thus, the group generated by cocycles unramified outside $S$ classifies abelian extensions of $k$ with degree bounded by the order of $P^{D}(1)$ which are unramified outside $S$. That the number of such extensions is finite is a well-known result due to Hermite and Minkowski.

Acknowledgements. The author would like to thank M. Hochster, W. Raskind, and P. Salberger for their help, and would like to convey special thanks to J.-L. Colliot-Thélène for his extraordinary investment of time and energy in reading and commenting on earlier versions of this manuscript.

\section{References}

1. Barlow, R.: Rational equivalence of zero cycles for some more surfaces with $p_{g}=0$. Invent. Math. 79, 303-308 (1985)

2. Bloch, $\mathrm{S}$.: $K_{2}$ of artinian Q-algebras, with application to algebraic cycles. Commun. Algebra 3, 405-428 (1975)

3. Bloch, S.: Lectures on algebraic cycles. (Duke Univ. Math. Ser. vol. IV) Durham, NC: Duke University Press 1980

4. Bloch, S.: On the Chow groups of certain rational surfaces. Ann. Sci. Éc. Norm. Supér. 14, 41-59 (1981)

5. Bloch, S., Kas, A., Lieberman, D.: Zero cycles on surfaces with $p_{g}=0$. Compos. Math. 33, 135-145 (1976)

6. Borel, A., Serre, J.-P.: Le théorème de Riemann-Roch. Bull. Soc. Math. Fr. 86, 97-136 (1958)

7. Colliot-Thélène, J.-L.: Hilbert's theorem 90 for $K_{2}$, with application to the Chow groups of rational surfaces. Invent. Math. 71, 1-20 (1983)

8. Colliot-Thélène, J.-L., Ischebeck, F.: L'équivalence rationnelle sur les cycles de dimension zéro des variétés algébriques réelles. C.R. Acad. Sci., Paris 292, 723-725 (1981)

9. Colliot-Thélène, J.-L., Parimala, R.: Real connected components of algebraic varieties and étale cohomology (Preprint)

10. Colliot-Thélène, J.-L., Raskind, W.: $K_{2}$-cohomology and the second Chow group. Math. Ann. 270, 165-199 (1985)

11. Colliot-Thélène, J.-L., Raskind, W.: On the reciprocity law for surfaces over finite fields. J. Fac. Sci., Univ. Tokyo, Sect. IA 33, 283-294 (1986)

12. Colliot-Thélène, J.-L., Sansuc, J.-J.: On the Chow groups of certain rational surfaces: a sequel to a paper of S. Bloch. Duke Math. J. 48, 421-447 (1981)

13. Colliot-Thélène, J.-L., Sansuc, J.-J., Soulé, C.: Torsion dans le groupe de Chow de codimension deux. Duke Math. J. 50, 763-801 (1983)

14. Coombes, K.R.: The arithmetic of Enriques surfaces (Preprint)

15. Coombes, K.R., Muder, D.J.: Zero cycles on del Pezzo surfaces over local fields. J. Algebra 97, 438-460 (1985)

16. Cossec, F., Dolgachev, I.: Enriques surfaces. Boston: Birkhäuser 1988

17. Gillet, H.: $K$-theory and intersection theory revisited. $K$-Theory 1, $405-415$ (1987)

18. Grayson, D.: Products in $K$-theory and intersecting algebraic cycles. Invent. Math. 47, 71-83 (1978) 
19. Gros, M.: 0-cycles de degré 0 sur les surfaces fibrées en coniques. J. Reine Angew. Math. 373, 166-184 (1987)

20. Gros, M., Suwa, N.: Application d'Abel-Jacobi p-adique et cycles algébriques. Duke Math. J. 57, 579-613 (1988)

21. Grothendieck, A.: Géométrie formelle et géométrie algébrique. Séminaire Bourbaki, Exposé $182(1958 / 59)$

22. Hartshorne, R.: Algebraic geometry. Berlin Heidelberg New York: Springer 1977

23. Inose, H., Mizukami, M.: Rational equivalence of 0-cycles on some surfaces of general type with $p_{g}=0$. Math. Ann. 244, 205-217 (1979)

24. Kato, K., Saito, S.: Unramified class field theory of arithmetical surfaces. Ann. Math. 118, 241-275 (1983)

25. Keum, J.H.: On Kummer surfaces. Ph. D. thesis, University of Michigan 1988

26. Merkurjev, A.S., Suslin, A.A.: K-cohomology of Severi-Brauer varieties and norm residue homomorphism. Izv. Akad. Nauk. SSSR, Ser. Mat. 46, 1011-1046 (1982); English translation in Math. USSR, Izv. 21, 307-341 (1983)

27. Muder, D.J.: Concerning a conjecture of Colliot-Thélène and Sansuc. Duke Math. J. 55, $51-63(1987)$

28. Murthy, M.P., Roy, A.: Torsion in $K_{2}$ of fields and 0-cycles on rational surfaces. Comment. Math. Helv. 59, 165-186 (1984)

29. Quillen, D.: Higher algebraic $K$-theory I. In: Bass, H. (ed.) Algebraic $K$-theory I. (Lect. Notes Math., vol. 341, pp. 85-147) Berlin Heidelberg New York: Springer 1973

30. Raskind, W.: 0-cycles on surfaces with $p_{g}=q=0$ (Preprint)

31. Raskind, W.: Torsion algebraic cycles on varieties over local fields. In: Proceedings of the Lake Louise K-Theory Conference. Dordrecht: D. Reidel 1989

32. Salberger, P.: Zero-cycles on rational surfaces over number fields. Invent. Math. 91, 505-524 (1988)

33. Serre, J.-P.: Local fields. Berlin Heidelberg New York: Springer 1979

34. Shatz, S.S.: Profinite groups, arithmetic, and geometry. (Ann. Math. Stud., vol. 67) Princeton: Princeton University Press 1972

35. Sherman, C.C.: Some theorems on the $K$-theory of coherent sheaves. Commun. Algebra 7 , 1489-1508 (1979)

36. Suslin, A.A.: Torsion in $K_{2}$ of fields. $K$-Theory 1, 5-29 (1987)

37. Tate, J.: Duality theorems in Galois cohomology over number fields. In: Proc. ICM, Stockholm, pp. 234-241, 1962. Djursholm: Institute Mittag-Leffler 1963

38. Tate, J.: The cohomology groups of tori in finite Galois extensions of number fields. Nagoya Math. J. 27, 709-719 (1966)

39. Waldhausen, F.: Algebraic $K$-theory of generalized free products. Ann. Math. 108, 135-256 (1978) 